\title{
Topisch appliziertes L-Argininhydrochlorid verbessert Hauttrockenheit und Pruritus bei Patienten unter chronischer Hämodialyse - eine Vehikel- kontrollierte prospektive randomisierte Studie im Halbseitenvergleich
}

\author{
Topical L-Arginine Hydrochloride Ointment Improves Skin Dryness and Pruritus in Patients under Chronic \\ Haemodialysis - A Vehicle-Controlled Prospective Randomised Study
}

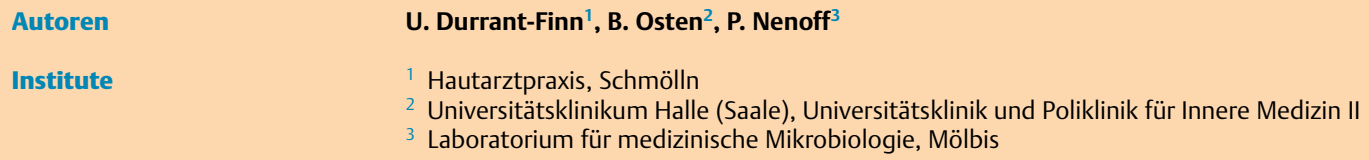

\section{Bibliografie}

DOI $10.1055 / \mathrm{s}-2008-1077317$

Akt Dermatol 2008; 34:

175-187 @ Georg Thieme

Verlag KG Stuttgart · New York ISSN 0340-2541

\section{Korrespondenzadresse}

Prof. Dr. med. Pietro Nenoff

Haut- und Laborarzt/

Allergologie

Laboratorium für medizinische

Mikrobiologie

Straße des Friedens 8

04579 Mölbis

nenoff@mykologie-experten.de

\section{Zusammenfassung}

Hintergrund: Der urämische Pruritus ist ein belastendes Symptom bei Hämodialysepatienten, von denen 50 bis $90 \%$ betroffen sind. Die Ursachen des urämischen Juckreizes sind bisher nicht eindeutig aufgedeckt. Beim Fehlen einer kausalen Therapie lässt sich der urämische Pruritus letztlich nur durch eine Nierentransplantation effektiv behandeln.

Patienten und Methoden: 24 Hämodialysepatienten und 12 hautgesunde Kontrollpersonen wurden in einem Halbseitenvergleich in einer randomisierten Vehikel-kontrollierten Studie über einen Zeitraum von 4 Wochen mit einer 2,5\%igen L-Arginin-Salbe (Wirkstoff) und einer wirkstofffreien Salbe (Vehikel) behandelt.

Ergebnisse: Die Hauttrockenheit ließ sich bei den Hämodialysepatienten sowohl mit dem Wirkstoff als auch Vehikel statistisch signifikant verbessern. Topisches L-Arginin führte bei den

\section{Einleitung}

$\nabla$

Die Hautveränderungen unter Dialyse bei chronisch-terminaler Niereninsuffizienz sind vielfältig [1]. Sie umfassen die Hautalterung und Xerosis, Gefäßveränderungen, Porphyria cutanea tarda (Pseudoporphyrie), Verschlechterung einer Psoriasis vulgaris, Infektionen, perforierende Dermatosen, Pigment- und Nagelveränderungen, metastatische Kalzinose, kutane Oxalose, Präkanzerosen der Haut sowie manifeste Hauttumoren, und den urämischen Pruritus, welcher ca. 50-90\% der chronischen Dialysepatienten betrifft [2,3]. Die kausale Genese des urämischen Pruritus ist bis jetzt nicht geklärt. In den letzten Jahren wurden fünf pathophysiologische Hypothesen favorisiert: fördernde Einflüsse (z.B. Kalziumphosphatablagerungen in der Epidermis), Stimuli (z.B. sekundärer Hyperparathyreoidis-
Hämodialysepatienten zu einer signifikanten Besserung des urämischen Pruritus. Darüber hinaus ließen sich Erythem und Schuppung mit dem Wirkstoff signifikant bessern. Die Corneometrie erbrachte zum Zeitpunkt 0 für die Hämodialysepatienten einen deutlich niedrigeren Wert, nach vier Wochen Behandlung mit dem Wirkstoff lag eine höhere Hautfeuchtigkeit vor. Vier Wochen Behandlung mit Wirkstoff und Vehikel hatte keinen Einfluss auf den transepidermalen Wasserverlust (TEWL). Kutane oder systemische Nebenwirkungen der L-Arginin-Behandlung traten nicht auf.

Schlussfolgerungen: Der positive Effekt von 2,5\% L-Argininhydrochlorid-Salbe auf den urämischen Pruritus kann hypothetisch damit erklärt werden, dass L-Arginin als Substrat der Stickoxidsynthase (NOS)-Isoformen die immunmodulierenden, antimikrobiellen und vasodilatativen Eigenschaften von Stickoxid (NO) wirksamer werden lässt.

mus), neuropathische Störungen (z.B. anomale kutane Innervation bei urämischer peripherer Neuropathie) sowie zentralnervöse Veränderungen (z. B. Kumulation von Endorphinen beim Urämiker mit Verstärkung des Pruritus) und immunologische Vorgänge $[4,5]$. Die Immunhypothese gewinnt zunehmend an Bedeutung, da eine Besserung des urämischen Pruritus z.B. durch den topischen Calcineurininhibitor Tacrolimus zu beobachten ist. Symptomatische Therapien sind in den AWMF-Leitlinien zum Pruritus zu finden. Dennoch sistiert der urämische Pruritus letztlich nur durch eine Nierentransplantation. Die derzeitigen topischen und systemischen Therapiemöglichkeiten beim urämischen Pruritus sind deshalb symptomatisch bzw. polypragmatisch. Für die Hydratation der Haut hat das „Feuchthalte-Komplex-System“ eine große Bedeutung. Harnstoff ist einer der wichtigsten natürlichen 
Feuchthaltefaktoren (natural moisturizing factors). Harnstoffuntersuchungen des Stratum corneum ergaben, dass ältere Menschen einen erniedrigten, Urämiker jedoch - trotz Trockenheit - paradoxerweise einen erhöhten Harnstoffwert haben (Normwert bei Hautgesunden um $25 \mu \mathrm{g} / 2,5 \mathrm{~cm}^{2}$ ). Die Bedeutung des Harnstoffs im „Feuchthalte-Komplex-System“ der urämischen Haut ist deshalb nicht geklärt. Aktuelle Untersuchungen zeigen eine signifikante Wirkung von topisch applizierter 2,5\%iger L-Arginin-Salbe, einer semiessenziellen Aminosäure, auf Hauttrockenheit und Pruritus bei Patienten mit atopischem Ekzem und Altershaut [6].

In dieser Vehikel-kontrollierten, offenen Studie sollte untersucht werden, wie die topische Applikation von L-Arginin bei Dialysepatienten im „Feuchthalte-Komplex-System“ der Haut in vivo wirkt. Der Einfluss der L-Arginin-haltigen Präparation auf die klinischen Parameter Hauttrockenheit, Juckreiz, Erythem und Schuppung wurde bestimmt, darüber hinaus auch die Hautfeuchtigkeit mittels Corneometrie sowie der transepidermale Wasserverlust (TEWL) mittels Tewametrie gemessen.

\section{Patienten und Methoden \\ $\nabla$}

\section{Studiendesign}

Die Untersuchungen erstreckten sich auf einen Zeitraum von Dezember 2002 bis März 2003. Einbezogen wurden 24 Patienten unter chronischer Hämodialyse (PHV-Dialysezentrum Altenburg, Kreuzstraße 5, 04600 Altenburg, Leitung: Frau Dres. Weber/Graupner) und 12 haut- und nierenfunktionsgesunde Kontrollpersonen ( Tab. 1-4). Es kamen High-flux- $(n=23)$ und Low-flux-Dialysatoren $(\mathrm{n}=1)$ zum Einsatz.

Tab. 1 Altersverteilung der Patienten $(n=24)$ und Kontrollpersonen $(n=12)$

\begin{tabular}{lll} 
& Hämodialysepatienten & Kontrollpersonen \\
Männlich & $52-82$ Jahre $(n=13)$ & $37-84$ Jahre $(n=6)$ \\
\hline Weiblich & $29-74$ Jahre $(n=11)$ & $35-70$ Jahre $(n=6)$ \\
\hline Mittelwert & $60,04 \pm 12,2$ & $56,17 \pm 17,6$
\end{tabular}

Bei beiden Gruppen erfolgten die Untersuchungen in 4 Visiten über einen Zeitraum von 6 Wochen. Alle Patienten und Probanden wurden über einen Zeitraum von 4 Wochen mit einer 2,5\%igen L-Argininhydrochlorid-Salbe und der wirkstofffreien Salbe behandelt.

Die Untersuchungen wurden in Übereinstimmung mit der Deklaration von Helsinki von 1975 in der Überarbeitung von 1983 durchgeführt. Die Patienten wurden vor Beginn der Studie ausführlich über deren Inhalt und Ziele informiert. Die Teilnahme erfolgte freiwillig und auf der Basis einer schriftlichen Einverständniserklärung unter Berücksichtigung der folgenden Einund Ausschlusskriterien.

\section{Einschlusskriterien}

$\checkmark$ mindestens 18 Jahre alt

- vorliegende Einverständniserklärung

- Diagnosen: terminale Niereninsuffzienz - dialysepflichtig gesunde Patienten als Vergleichsgruppe
Tab. 2 Hämodialysedauer zum Zeitpunkt der Untersuchung in Jahren $(n=24)$

\begin{tabular}{lclll|} 
& $\mathbf{5}$ Jahre & $\begin{array}{l}\mathbf{6 - 1 0} \\
\text { Jahre }\end{array}$ & $\begin{array}{l}\mathbf{1 1 - 1 7} \\
\text { Jahre }\end{array}$ & Gesamt \\
Männlich & 6 & 6 & 1 & 13 \\
\hline Weiblich & 8 & 3 & & 11 \\
\hline Gesamt & 14 & 9 & 1 & 24 \\
\hline
\end{tabular}

\begin{tabular}{|c|c|c|c|c|c|}
\hline \multirow[t]{2}{*}{$3 \times /$ Woche } & 4 & 4,30 & 5 & 5,30 & \multirow[t]{2}{*}{ Gesamt } \\
\hline & \multicolumn{4}{|c|}{ Stunden } & \\
\hline Männer & 3 & 4 & 5 & 1 & 13 \\
\hline Frauen & 5 & 4 & 2 & & 11 \\
\hline Gesamt & 8 & 8 & 7 & 1 & 24 \\
\hline
\end{tabular}

Tab. 4 Nephrologische Grunderkrankungen

\begin{tabular}{ll} 
Chronische Glomerulonephritis & $n=4$ \\
\hline Chronische Pyelonephritis & $n=4$ \\
Zystennieren & $n=4$ \\
Chronisch interstitielle Nephritis & $n=3$ \\
Nephrosklerose & $n=2$ \\
\hline Schrumpfnieren (ungeklärter Genese) beidseits & $n=2$ \\
\hline Schrumpfniere (ungeklärter Genese) bei Einnierigkeit & $n=1$
\end{tabular}

\section{Ausschlusskriterien \\ $\nabla$ \\ Diabetes mellitus \\ - akute entzündliche Dermatosen \\ - bekannte Allergien gegen einen oder mehrere Inhaltsstoffe des Präparates \\ - systemische Medikation oder lokale Therapie von Dermatosen \\ - Schwangerschaft und Stillzeit}

\section{Dermatologischer Befund $\nabla$}

Beide Untersuchungsgruppen wurden in Anlehnung an den Atopiescore nach Diepgen [7] dermatologisch beurteilt: Hauttrockenheit, Pruritus, Erythem und Schuppung.

\section{Wirkstoff- und Vehikelpräparation}

- Wirkstoffhaltige Salbe: 2,5\%ige L-Argininhydrochlorid-Salbe mit Salbengrundlage

- Cetylstearylalkohol $\quad 0,25$ Teile

- Wollwachsalkohol 3,00 Teile

- weiße Vaseline $\quad 46,75$ Teile

- gereinigtes Wasser 50,00 Teile

- Wirkstofffreie Salbe: Salbengrundlage w. o. ohne Wirkstoff 


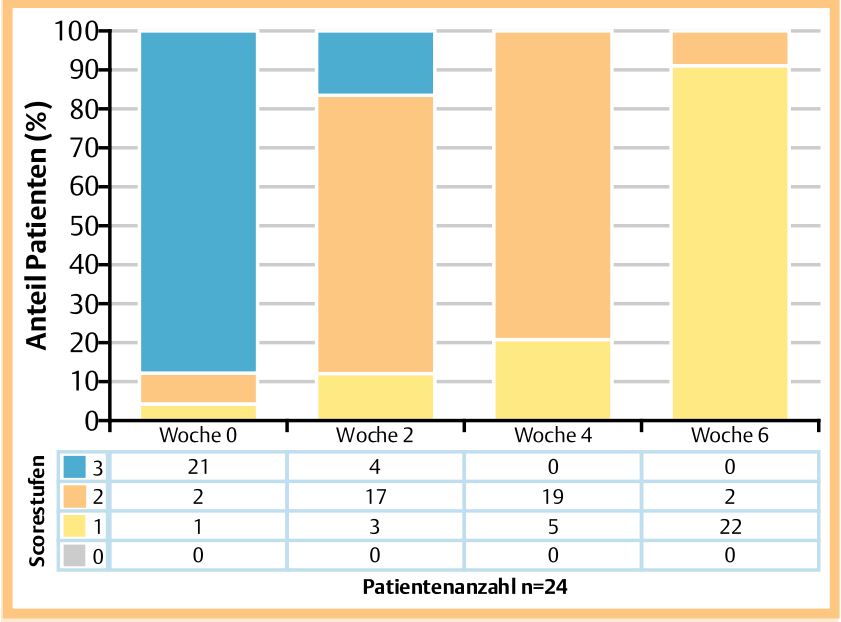

Abb. 1 a Einfluss der wirkstofffreien Salbe (Vehikel) auf die Hauttrockenheit bei Hämodialysepatienten $(n=24)$. Statistische Auswertung, Veränderungen im Symmetrietest, Wochenvergleich: Woche 0 bis 2: signifikant bei $\alpha=1 \%$ (Prüfgröße $=19,0 ; \chi^{2}$-Tafelwert $[1 \%, 6]=16,8$ ), Woche 2 bis 4 : nicht signifikant, Woche 4 bis 6 : signifikant bei $\alpha=1 \%$ (Prüfgröße $=17,0$; $\chi^{2}$-Tafelwert $[1 \%, 6]=16,8$ ), Woche 0 bis 6 : signifikant bei $\alpha=1 \%$ (Prüfgröße $=23,0 ; \chi^{2}$-Tafelwert $[1 \%, 6]=16,8$ ).

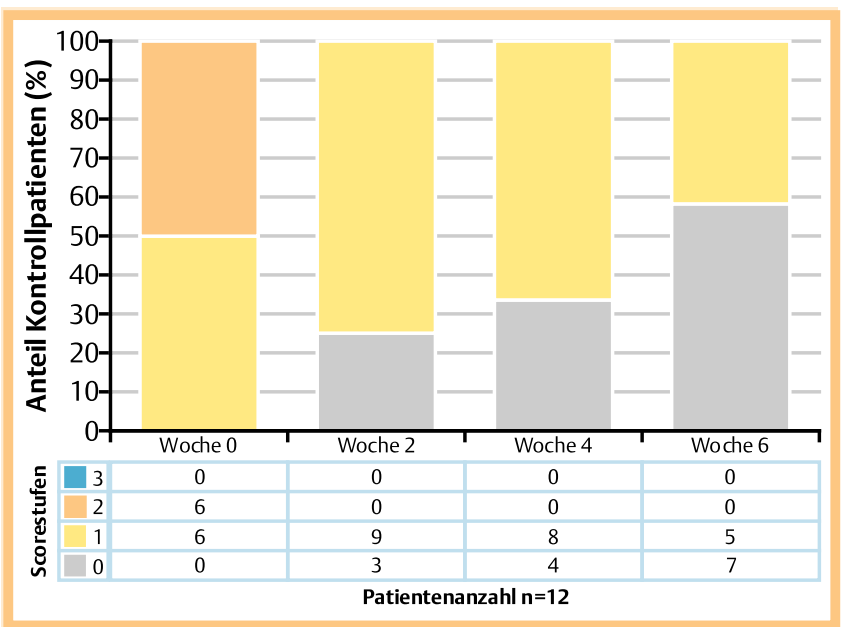

Abb. 1c Einfluss der wirkstofffreien Salbe (Vehikel) auf die Hauttrockenheit bei hautgesunden Kontrollpersonen $(n=12)$. Statistische Auswertung: Veränderungen im Symmetrietest, Wochenvergleich: Woche 0 bis 2 , 2 bis 4,4 bis 6 und 0 bis 6 : nicht signifikant.

\section{Studienablauf}

Im Rahmen der sechswöchigen Studie (vier Wochen Therapie, danach zwei Woche ohne Behandlung) wurden 4 Visiten vorgenommen. Der Zeitpunkt 0 stellte den Beginn der Untersuchungen (vor Therapie) dar, Zeitpunkt 2 folgte nach zwei, der Zeitpunkt 4 nach vier, der Zeitpunkt 6 nach sechs Wochen.

Für die Dokumentation des dermatologischen Befundes (Hauttrockenheit, Juckreiz, Erythem, Schuppung) der betroffenen Hautareale wurden vier Ausprägungsstufen unterschieden: $0=$ keine, 1 = gering, $2=$ mäßig und $3=$ stark.

Die Patienten erhielten die Präparate ohne Erkennbarkeit des Wirkstoffes im Sinne einer kontrollierten „blinden“ Studie. Laut Anweisung sollte die Salbe zweimal täglich auf die definierten Hautareale appliziert werden. Als Hautareale wurden der rechte und linke Unterarm volar definiert. Dabei wurde die wirkstoff-

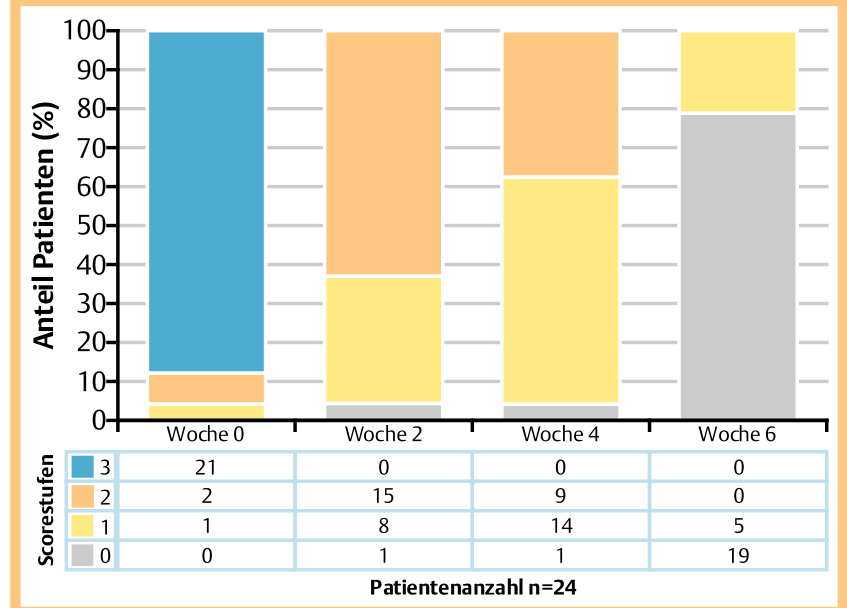

Abb. 1 b Einfluss der 2,5\%igen L-Arginin-Salbe (Wirkstoff) auf die Hauttrockenheit bei Hämodialysepatienten $(n=24)$. Statistische Auswertung, Veränderungen im Symmetrietest, Wochenvergleich: Woche 0 bis 2: signifikant bei $\alpha=1 \%$ (Prüfgröße $=24,0 ; \chi^{2}$-Tafelwert $[1 \%, 6]=16,8$ ), Woche 2 bis 4: nicht signifikant, Woche 4 bis 6 und 0 bis 6 : jeweils signifikant bei $\alpha=1 \%$ (Prüfgröße $=22,0 ; \chi^{2}$-Tafelwert $[1 \%, 6]=16,8$ ).

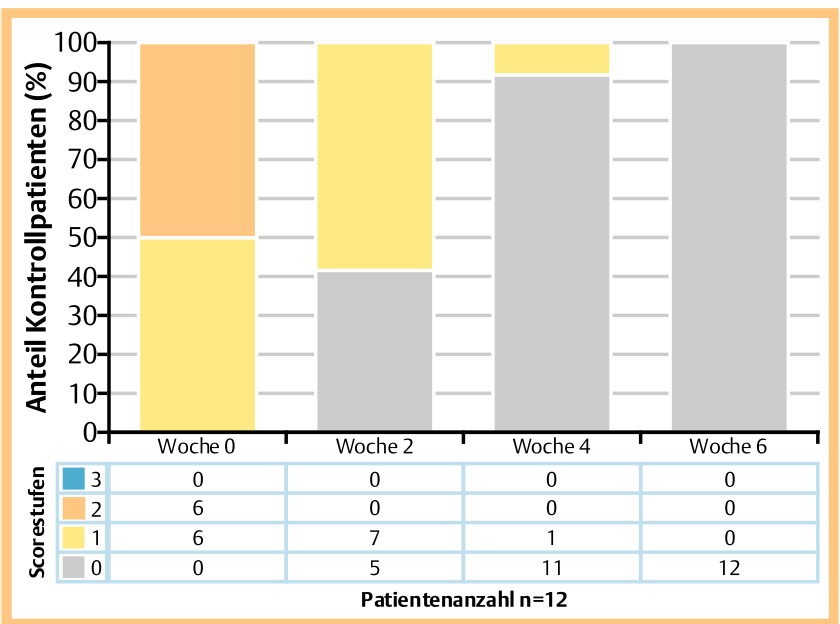

Abb. 1 d Einfluss der 2,5\%igen L-Arginin-Salbe (Wirkstoff) auf die Hauttrockenheit bei hautgesunden Kontrollpersonen $(n=12)$. Statistische Auswertung: Veränderungen im Symmetrietest, Wochenvergleich: Woche 0 bis 2,2 bis 4,4 bis 6 und 0 bis 6 : nicht signifikant.

haltige Salbe am linken Unterarm volar in einem Areal von $15 \times 15 \mathrm{~cm}$ zweimal täglich aufgetragen. Die wirkstofffreie Salbe wurde am rechten Unterarm volar in einem Areal von $15 \times 15 \mathrm{~cm}$ zweimal täglich aufgetragen. Beide Gruppen erhielten kodierte Prüfpräparate (ohne Erkennbarkeit des Wirkstoffes). In Woche 5 und 6 erfolgte keine Salbentherapie.

\section{Corneometrie}

Die Hautfeuchtigkeitsmessung erfolgte mit dem Corneometer CM 820 (Schwarzhaupt Medizintechnik GmbH, Köln). Bei jeder Untersuchung wurde der Mittelwert aus 10 Einzelmessungen, die an benachbarten Messstellen im Untersuchungsareal vorgenommen wurden, gebildet. Vor Beginn der Messungen wurden die Probanden auf einer Untersuchungsliege über ca. 10 Minuten akklimatisiert. 


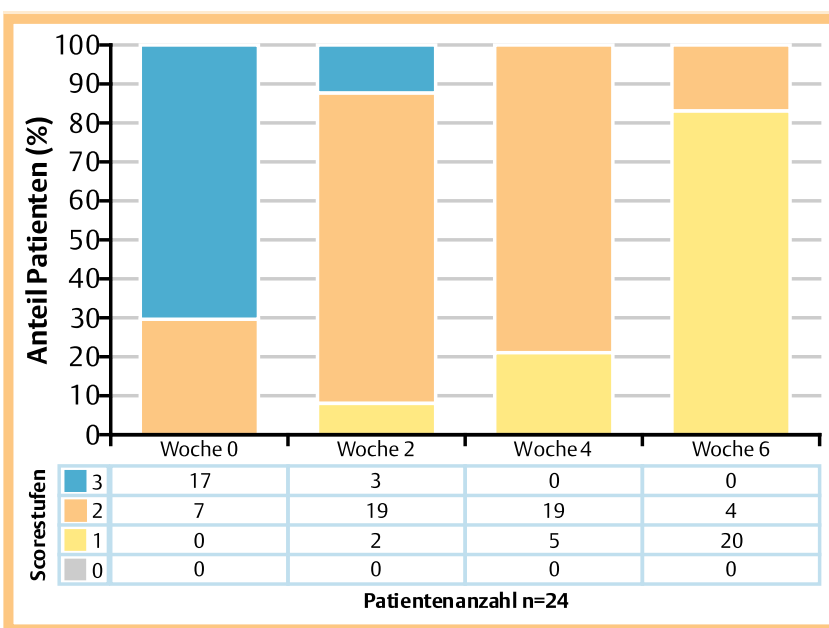

Abb. 2 a Einfluss der wirkstofffreien Salbe (Vehikel) auf den Juckreiz bei Hämodialysepatienten $(n=24)$. Statistische Auswertung: Veränderungen im Symmetrietest, Wochenvergleich: Woche 0 bis 2: signifikant bei $\alpha=5 \%$ (Prüfgröße $=16,0 ; \chi^{2}$-Tafelwert $[5 \%, 6]=12,6$ ), Woche 2 bis 4: nicht signifikant, Woche 4 bis 6: signifikant bei $\alpha=5 \%$ (Prüfgröße $=15,0 ; \chi^{2}$-Tafelwert $[5 \%, 6]=12,6$ ), Woche 0 bis 6: signifikant bei $\alpha=1 \%$ (Prüfgröße = 23,$0 ; \chi^{2}$-Tafelwert $[1 \%, 6]=16,8$ ).

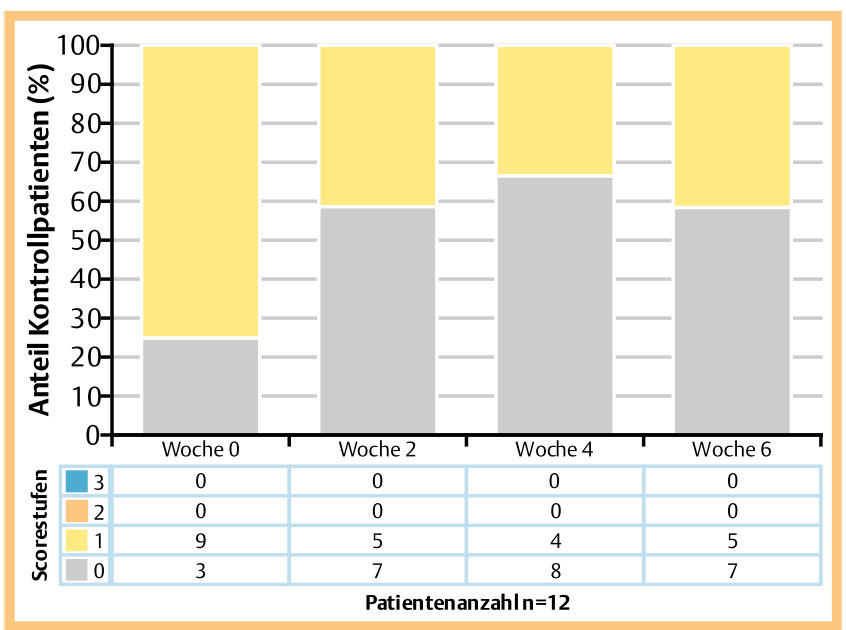

Abb.2c Einfluss der wirkstofffreien Salbe auf den Juckreiz bei hautgesunden Kontrollpersonen $(n=12)$. Statistische Auswertung: Veränderungen im Symmetrietest, Wochenvergleich: Woche 0 bis 2, 2 bis 4,4 bis 6 und 0 bis 6 : nicht signifikant.

Die Messwerte werden nicht mit einer Maßeinheit ausgegeben, sondern als Anzeigeeinheit (AE). Eine Anzeigeneinheit beträgt, bei einer Nominaleindringtiefe des Messfeldes von $20 \mu \mathrm{m}$, etwa 1,2 bis $1,3 \mathrm{mg}$ Wasser $/ \mathrm{cm}^{2}$. Dabei entsprachen $0,07 \mathrm{mg}$ Wasser einer Anzeigeeinheit des CM 820.

\section{Tewametrie}

Der TEWL wurde mit dem Tewameter TM 210 (Courage-Khazaka electronic GmbH, Köln) gemessen. Angegeben wird der TEWL der Haut in $\mathrm{g} / \mathrm{m}^{2} \times \mathrm{h}$.

\section{Statistik}

Die statistischen Berechnungen wurden mit dem Programm „Fragebogenauswertung und statistische Standardprüfverfahren“ durchgeführt. Für alle Signifikanzaussagen wurde eine maximale Irrtumswahrscheinlichkeit von $\alpha=5 \%$ zugelassen. „Signi-

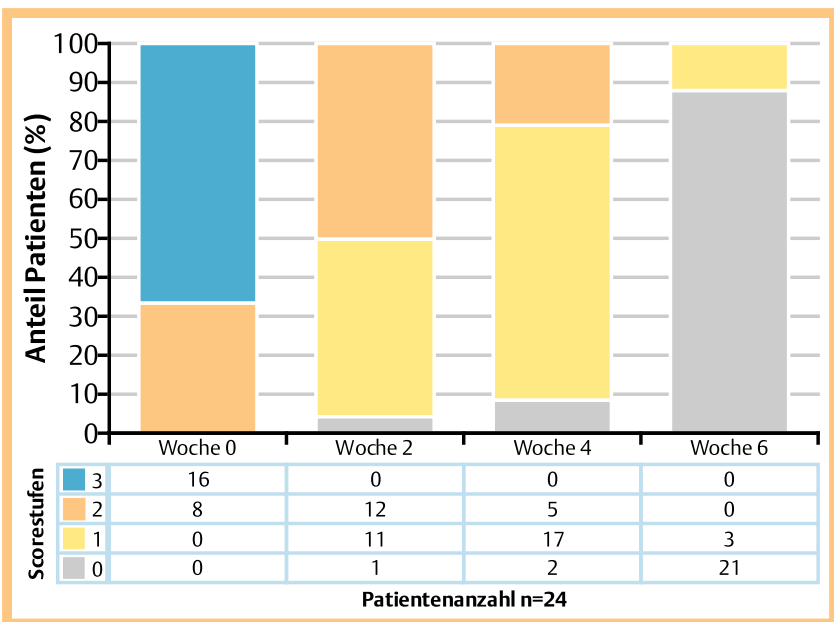

Abb. 2b Einfluss der 2,5\%igen L-Arginin-Salbe (Wirkstoff) auf den Juckreiz bei Hämodialysepatienten $(n=24)$. Statistische Auswertung: Veränderungen im Symmetrietest, Wochenvergleich: Woche 0 bis 2: signifikant bei $\alpha=1 \%$ (Prüfgröße $=24,0 ; \chi^{2}$-Tafelwert $[1 \%, 6]=16,8$ ), Woche 2 bis 4: nicht signifikant, Woche 4 bis 6 und 0 bis 6 : jeweils signifikant bei $\alpha=1 \%$ (Prüfgröße $=21,0 ; \chi^{2}$-Tafelwert $[1 \%, 6]=16,8$ ).

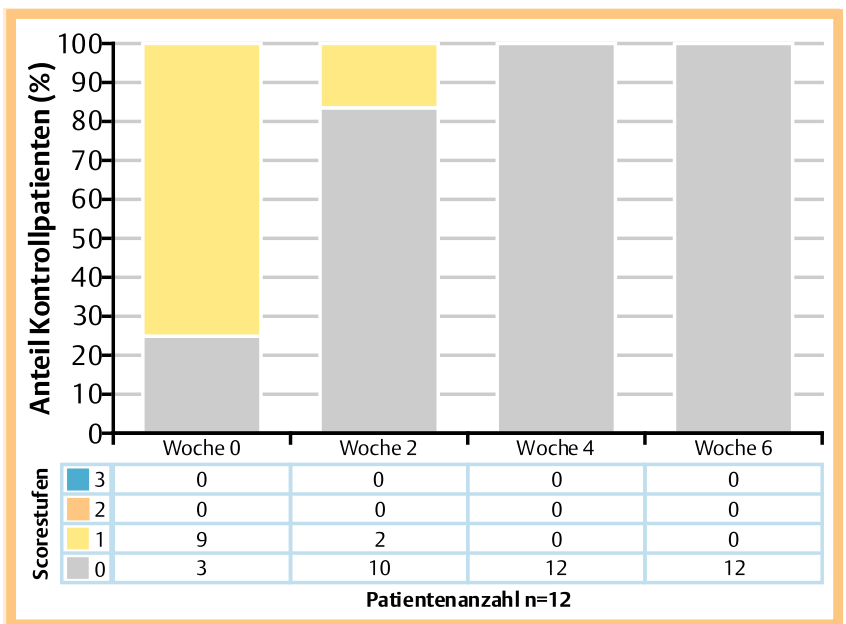

Abb. 2d Einfluss der der 2,5\%igen L-Arginin-Salbe (Wirkstoff) auf den Juckreiz bei hautgesunden Kontrollpersonen $(n=12)$. Statistische Auswertung: Veränderungen im Symmetrietest, Wochenvergleich: Woche 0 bis 2 , 2 bis 4,4 bis 6 und 0 bis 6 : nicht signifikant.

fikante Unterschiede“ zwischen zu vergleichenden Stichproben hinsichtlich der betrachteten Zielstellung sind echte (interpretierbare) Unterschiede, die nicht mehr zufälliger Natur sind.

\section{Ergebnisse}

$\nabla$

Einfluss wirkstofffreier und wirkstoffhaltiger Salbe auf Hauttrockenheit bei Hämodialysepatienten und Kontrollpersonen

Die Trockenheit der Haut hat sich unter der Behandlung und dann insbesondere von Woche 5 bis 6 auch unter der wirkstofffreien Salbe bei den Dialysepatienten signifikant verbessert ( $\bullet$ Abb. 1 a). Mit der wirkstoffhaltigen Salbe ließ sich die Hauttrockenheit jedoch im Vergleich zum Vehikel - trotz gleicher Signifikanz - über den Zeitraum Woche 0 bis 6 deutlich wirksa- 


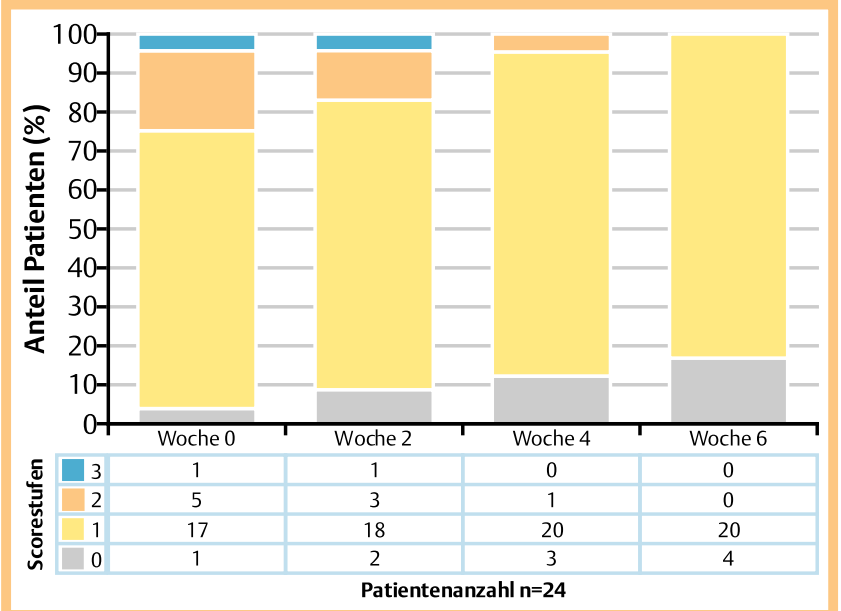

Abb. 3 a Einfluss der wirkstofffreien Salbe (Vehikel) auf das Erythem bei Hämodialysepatienten $(n=24)$. Statistische Auswertung: Veränderungen im Symmetrietest, Wochenvergleich: Woche 0 bis 2, 2 bis 4, 4 bis 6 und 0 bis 6: nicht signifikant.

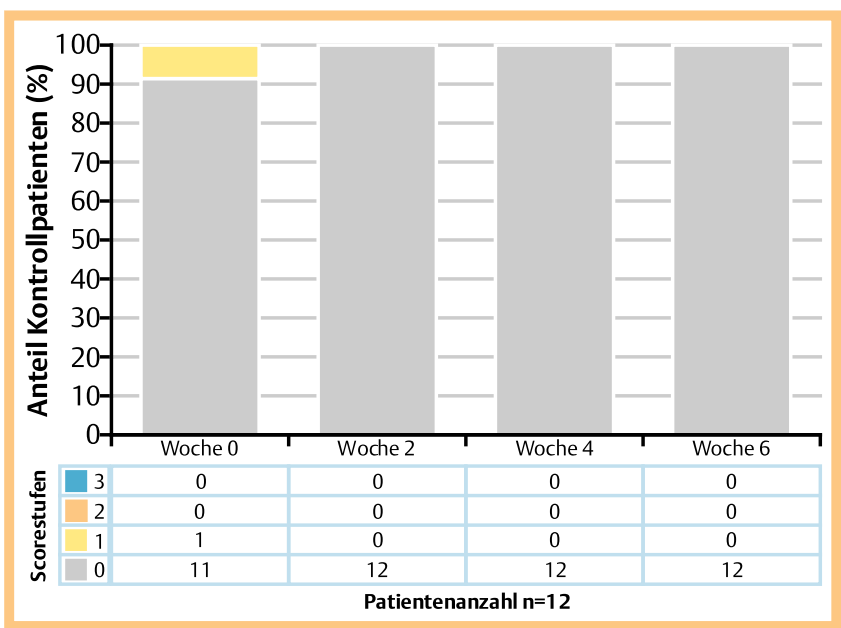

Abb.3c Einfluss der wirkstofffreien Salbe (Vehikel) auf das Erythem bei hautgesunden Kontrollpersonen $(n=12)$. Statistische Auswertung, Veränderungen im Symmetrietest: Woche 0 bis 2, 2 bis 4,4 bis 6 und 0 bis 6 : nicht signifikant.

mer beeinflussen. Bereits nach 2 Wochen war bei den Patienten keine starke Trockenheit mehr nachweisbar ( $\mathbf{A b b} \mathbf{1} \mathbf{b})$. Bei den hautgesunden Kontrollpersonen hatten weder Wirkstoff, noch Vehikel bei guter Ausgangssituation über den angegebenen Zeitraum einen signifikanten Effekt ( $\bullet$ Abb. 1 c u.d).

Einfluss wirkstofffreier und wirkstoffhaltiger Salbe auf den Juckreiz bei Hämodialysepatienten und Kontrollen Der Juckreiz besserte sich unter wirkstofffreier Salbe über den Behandlungszeitraum bei den Hämodialysepatienten signifikant, insbesondere auch nach Beendigung der Therapie in Woche 5 und 6 ( Abb.2a). Die wirkstoffhaltige Salbe hatte darüber hinausgehend einen deutlich signifikanten Effekt bei den Patienten ( Abb.2b). Zum Zeitpunkt Woche 6 gaben $87 \%$ der Dialysepatienten an, dass kein Juckreiz mehr bestand. In Bezug

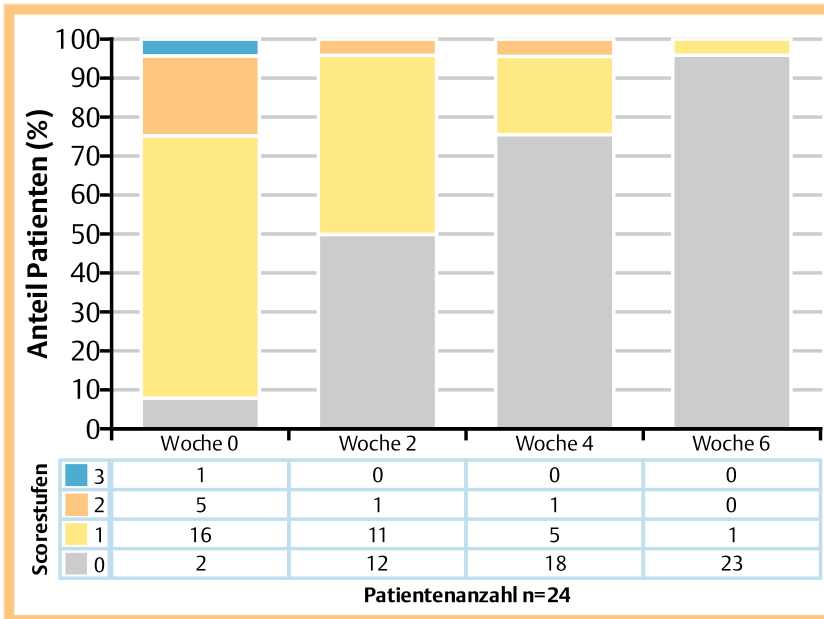

Abb. 3b Einfluss der 2,5\%igen L-Arginin-Salbe (Wirkstoff) auf das Erythem bei Hämodialysepatienten $(n=24)$. Statistische Auswertung, Veränderungen im Symmetrietest, Wochenvergleich: Woche 0 bis 2: signifikant bei $\alpha=5 \%$ (Prüfgröße $=15,0 ; \chi^{2}$-Tafelwert $[5 \%, 6]=12,6$ ), Woche 2 bis 4 und 4 bis 6 : nicht signifikant, Woche 0 bis 6 : signifikant bei $\alpha=1 \%$ (Prüfgröße $=22,0 ; \chi^{2}$-Tafelwert $\left.[1 \%, 6]=16,8\right)$.

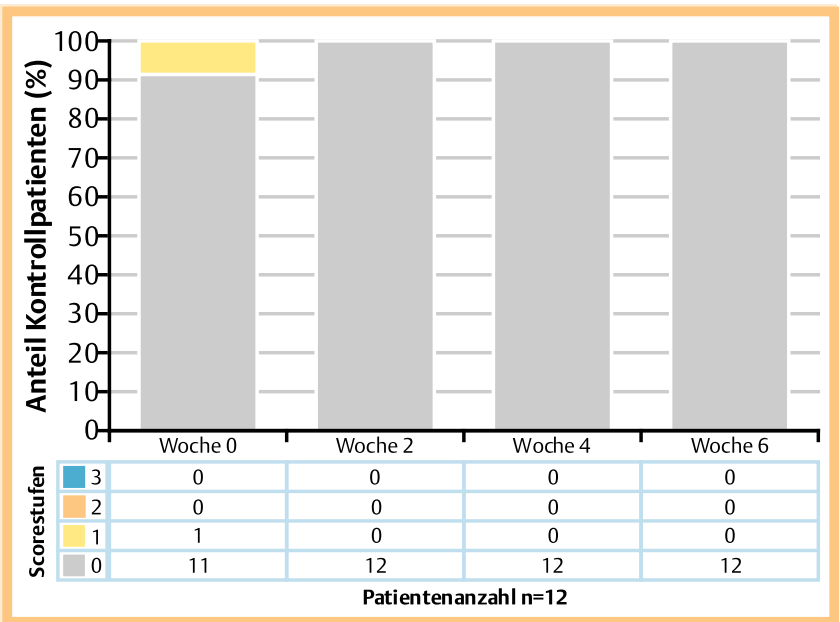

Abb. 3d Einfluss der 2,5\%igen L-Arginin-Salbe (Wirkstoff) auf das Erythem bei hautgesunden Kontrollpersonen $(n=12)$. Statistische Auswertung: Veränderungen im Symmetrietest, Wochenvergleich: Woche 0 bis 2 , 2 bis 4,4 bis 6 und 0 bis 6 : nicht signifikant.

auf den Juckreiz bei den Kontrollpersonen hatten Wirkstoff und Vehikel keinen signifikanten Effekt ( $\bullet$ Abb. 2cu.d).

Einfluss von Wirkstoff und Vehikel auf das Erythem bei Hämodialysepatienten und Kontrollen

Die wirkstofffreie Salbe hatte keinen Effekt auf das Symptom Erythem ( Abb. 3a). Dagegen ließ sich mit dem Wirkstoff eine signifikante Besserung des Erythems erzielen, besonders von Woche 0 bis 2 bzw. noch deutlicher von Woche 0 bis 6 ( Abb. 3b). In Bezug auf das Erythem hatten weder Wirkstoff noch Vehikel bei den hautgesunden Kontrollpersonen einen signifikanten Effekt ( $\bullet$ Abb.3cu.d). 


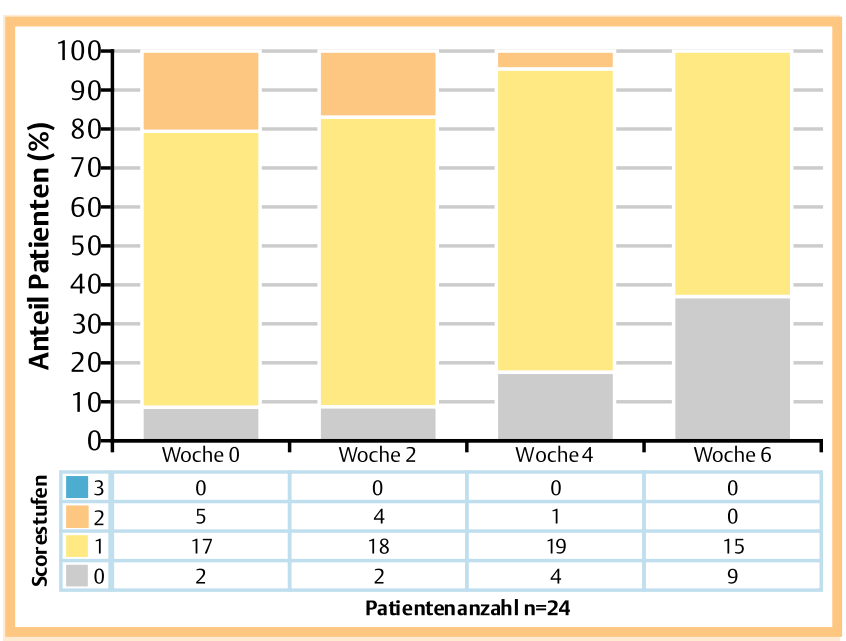

Abb. 4 a Einfluss der wirkstofffreien Salbe auf die Schuppung bei Hämodialysepatienten. Statistische Auswertung: Veränderungen im Symmetrietest, Wochenvergleich: Woche 0 bis 2, 2 bis 4,4 bis 6 und 0 bis 6 : nicht signifikant.

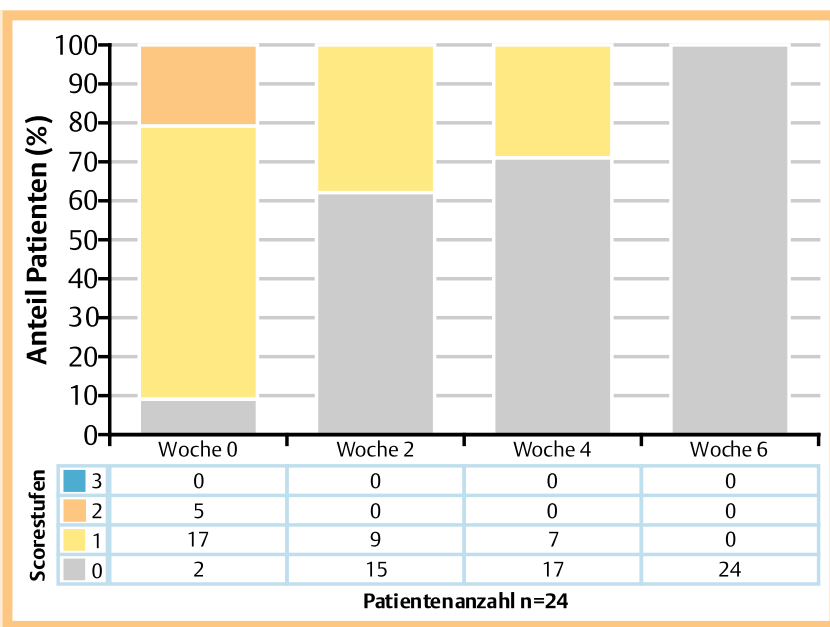

Abb. 4b Einfluss der 2,5\%igen L-Arginin-Salbe (Wirkstoff) auf die Schuppung bei Hämodialysepatienten $(n=24)$. Statistische Auswertung, Veränderungen im Symmetrietest, Wochenvergleich: Woche 0 bis 2: signifikant bei $\alpha=1 \%$ (Prüfgröße $=18,0 ; \chi^{2}$-Tafelwert $[1 \%, 6]=16,8$ ), Woche 2 bis 4 sowie 4 bis 6 : jeweils nicht signifikant, Woche 0 bis 6 : signifikant bei $\alpha=1 \%$ (Prüfgröße $=22,0 ; \chi^{2}$-Tafelwert $[1 \%, 6]=16,8$ )

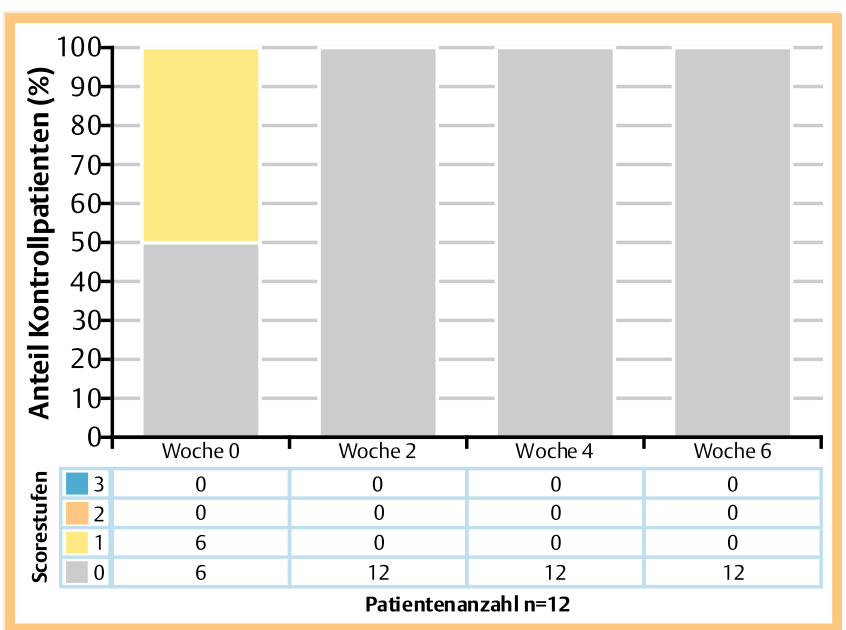

Abb. 4c Einfluss der wirkstofffreien Salbe (Vehikel) auf die Schuppung bei hautgesunden Kontrollpersonen $(n=12)$. Statistische Auswertung, Veränderungen im Symmetrietest: Woche 0 bis 2, 2 bis 4,4 bis 6 und 0 bis 6: nicht signifikant.

Einfluss wirkstofffreier und wirkstoffhaltiger Salbe auf die Hautschuppung bei Hämodialysepatienten und Kontrollpersonen

Auf die Hautschuppung der Hämodialysepatienten hatte die wirkstofffreie Salbe über den Behandlungszeitraum keinen signifikanten Effekt ( $\bullet$ Abb. 4 a). Dagegen zeigte die wirkstoffhaltige Präparation von 2,5\% L-Arginin einen signifikanten Effekt auf die Schuppung der Patienten, besonders auch nach Absetzen der Salbe bis zur Woche 6 ( $\bullet$ Abb. 4b). In Bezug auf die Schuppung hatten weder Wirkstoff noch Vehikel bei den hautgesunden Kontrollpersonen einen signifikanten Effekt ( $\bullet$ Abb. 4c u.d).

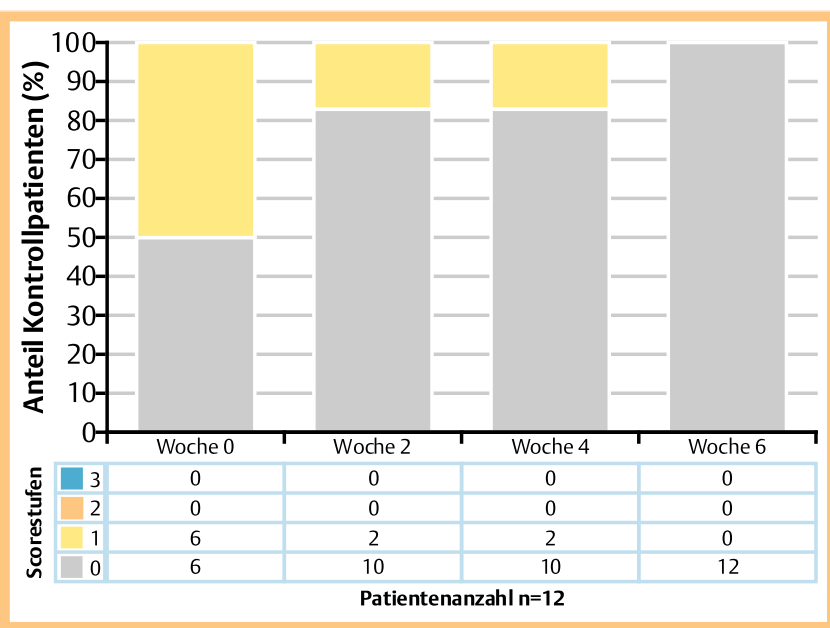

Abb. 4d Einfluss 2,5\%iger L-Arginin-Salbe (Wirkstoff) auf die Schuppung bei hautgesunden Kontrollpersonen $(n=12)$. Statistische Auswertung, Veränderungen im Symmetrietest: Woche 0 bis 2, 2 bis 4,4 bis 6 und 0 bis 6: nicht signifikant.

Der Einfluss wirkstofffreier und -haltiger Salbe auf die Hautfeuchtigkeit bei Hämodialysepatienten und Kontrollpersonen

Die Hautfeuchtigkeit war bei den Hämodialysepatienten vor Therapie in Woche 0 mit $62,64 \mathrm{mg} / \mathrm{H}_{2} 0 / \mathrm{cm}^{3}$ deutlich niedriger im Vergleich zu den Kontrollpersonen mit $68,14 \mathrm{mg} / \mathrm{H}_{2} \mathrm{O} / \mathrm{cm}^{3}$ (O Abb. 5 a u.b).

Mittels Corneometrie war bei den Hämodialysepatienten nach Behandlung mit dem Wirkstoff L-Arginin in Woche 6 ein signifikanter Abfall auf $60,19 \mathrm{mg} / \mathrm{H}_{2} \mathrm{O} / \mathrm{cm}^{3}$ zu verzeichnen, unter Vehikel immerhin auch auf $65,88 \mathrm{mg} / \mathrm{H}_{2} \mathrm{O} / \mathrm{cm}^{3}$, was ebenfalls signifikant war $(\bullet$ Abb. 5 a). Von Woche 0 bis 4 stiegen die Messwerte unter Behandlung mit dem Wirkstoff von 62,55 auf $71,99 \mathrm{mg} /$ $\mathrm{H}_{2} \mathrm{O} / \mathrm{cm}^{3}$, unter Vehikel von 62,24 auf $69,46 \mathrm{mg} / \mathrm{H}_{2} \mathrm{O} / \mathrm{cm}^{3}$. Das spricht für einen stärkeren Effekt des Wirkstoffs auf die Erhöhung der Hautfeuchtigkeit. 


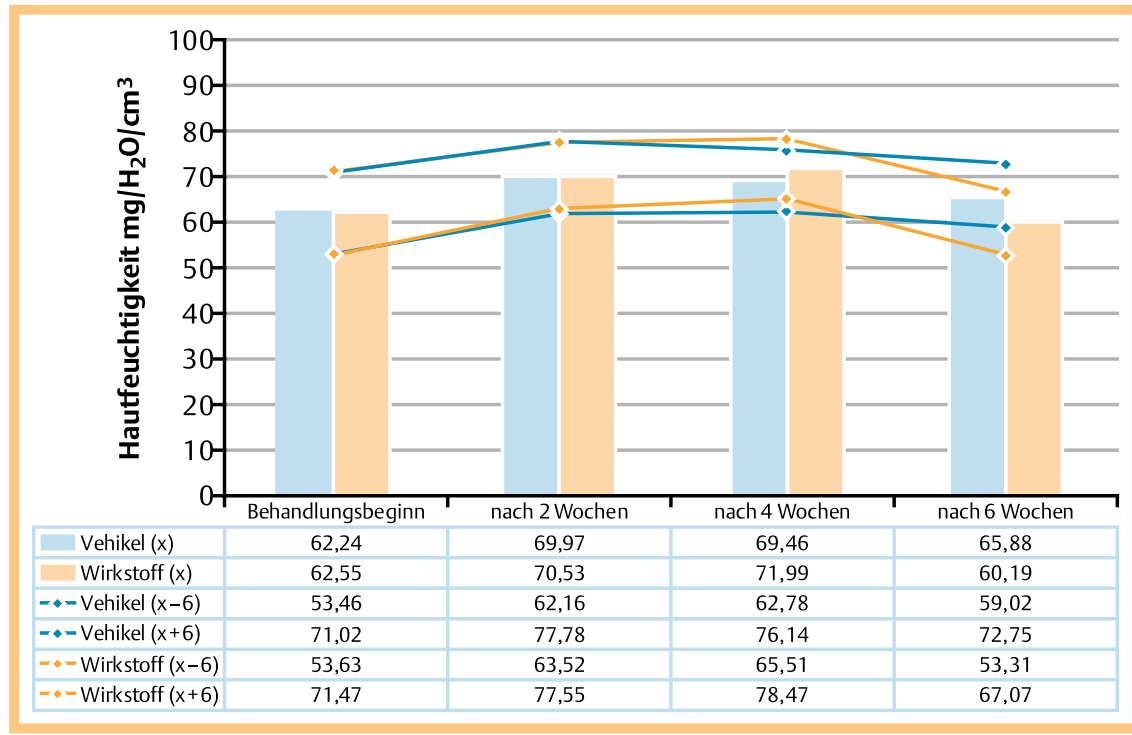

Abb. 5 a Einfluss wirkstofffreier Salbe (Vehikel) und 2,5\%iger L-Arginin-Salbe (Wirkstoff) auf die Hautfeuchtigkeit (Messung mittels Corneometrie) bei Hämodialysepatienten $(n=24)$. T-Test für Differenzen gegen Null, Veränderungen im Wochenvergleich (T-Test für Differenzen gegen Null): Woche 0 , 2 sowie 4: nicht signifikant. Woche 6 : signifikant bei $\alpha=1 \%$ (Prüfgröße $=3,79$; T-Tafelwert [1\%; $23]=2,81)$.

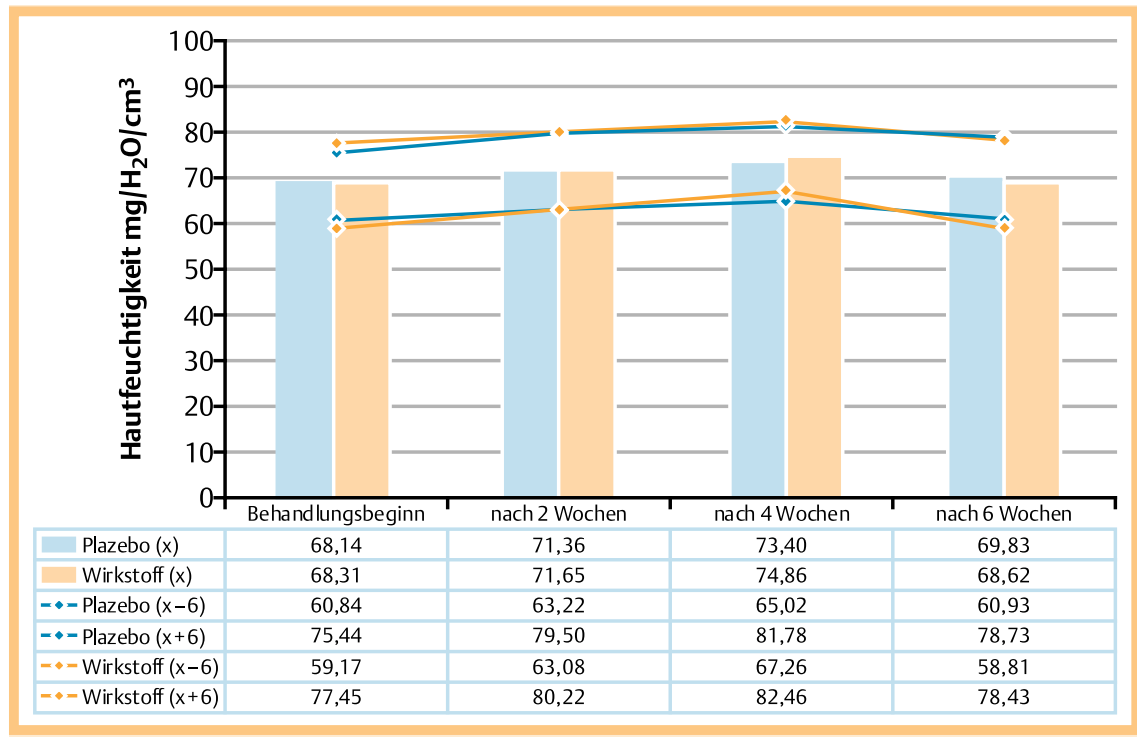

Abb. 5b Der Einfluss wirkstofffreier Salbe (Vehikel) und 2,5\%iger L-Arginin-Salbe (Wirkstoff) auf die Hautfeuchtigkeit (Messung mittels Corneometrie) bei Kontrollpersonen $(n=12)$. Veränderungen im Wochenvergleich (T-Test für Differenzen gegen Null): Woche 0, 2, 4 sowie 6: nicht signifikant.

Der Vergleich zwischen Wirkstoff und Vehikel ergab in der Gruppe der hautgesunden Kontrollen keinen signifikanten Unterschied, unter Wirkstoffbehandlung stieg die Hautfeuchtigkeit von Woche 0 bis 4 von 68,31 auf $74,86 \mathrm{mg} / \mathrm{H}_{2} \mathrm{O} / \mathrm{cm}^{3}$, unter Vehikel von 68,14 auf $73,40 \mathrm{mg} / \mathrm{H}_{2} \mathrm{O} / \mathrm{cm}^{3}$. Tendenziell hatte der Wirkstoff eine stärkere Wirkung auf die Erhöhung der Hautfeuchtigkeit bis zur Woche 4 ( $\bullet$ Abb. 5 b). Vehikel hatte zu keinem Zeitpunkt einen signifikanten Effekt auf die Hautfeuchtigkeit.

Einfluss von Wirkstoff und Vehikel auf den transepidermalen Wasserverlust (TEWL) bei Hämodialysepatienten und Kontrollpersonen

Der TEWL war zum Zeitpunkt der Woche 0 bei den Hämodialysepatienten signifikant niedriger mit $1,27 \mathrm{~g} / \mathrm{m}^{2} \times \mathrm{h}$, als bei den Kontrollpersonen mit $2,08 \mathrm{~g} / \mathrm{m}^{2} \times \mathrm{h}(\bullet$ Abb.6au.b). L-ArgininSalbe und die wirkstofffreie Salbe (Vehikel) bewirkten keine signifikanten Änderungen des TEWL bei den Hämodialysepatienten. Eine tendenzielle Senkung des TEWL unter Wirkstoff und Vehikel war zwischen Woche 2 und 4 zu verzeichnen. In Woche 6 , zwei Wochen nach Beendigung der externen Therapie mit
L-Arginin oder Vehikel, zeigte sich ebenfalls eine tendenzielle Erhöhung des TEWL in beiden Gruppen ( $\bullet$ Abb. 6 a). Auch bei den Kontrollen ergaben sich keine signifikanten Veränderungen durch die Wirkstoff- bzw. Vehikel-Behandlung.

Die Vehikel-Behandlung führte weder bei Dialysepatienten noch bei Kontrollen zu einer signifikanten Veränderung des TEWL. Zum Zeitpunkt Woche 2 und 4 war der TEWL bei den Hämodialysepatienten signifikant niedriger als bei den Kontrollpersonen (ه Abb.6b).

Nebenwirkungen/Unverträglichkeiten

Weder die 2,5\%ige L-Argininhydrochlorid-Salbe noch die Vehikelpräparation hatten irgendwelche kutane oder systemische Nebenwirkungen. 


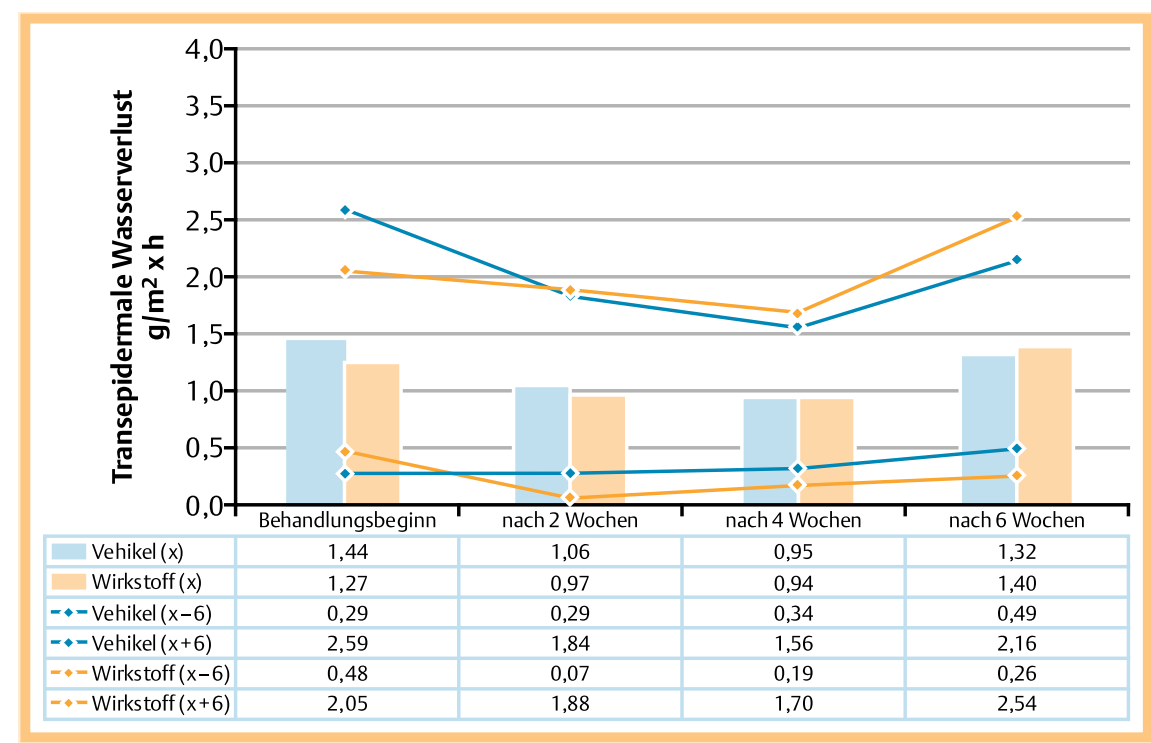

Abb. 6a Der Einfluss wirkstofffreier Salbe (Vehikel) und 2,5\%iger L-Arginin-Salbe (Wirkstoff) auf den transepidermalen Wasserverlust (TEWL) bei Hämodialysepatienten $(n=24)$. Veränderungen im Wochenvergleich (T-Test für Differenzen gegen Null): Woche 0, 2, 4 sowie 6: nicht signifikant.

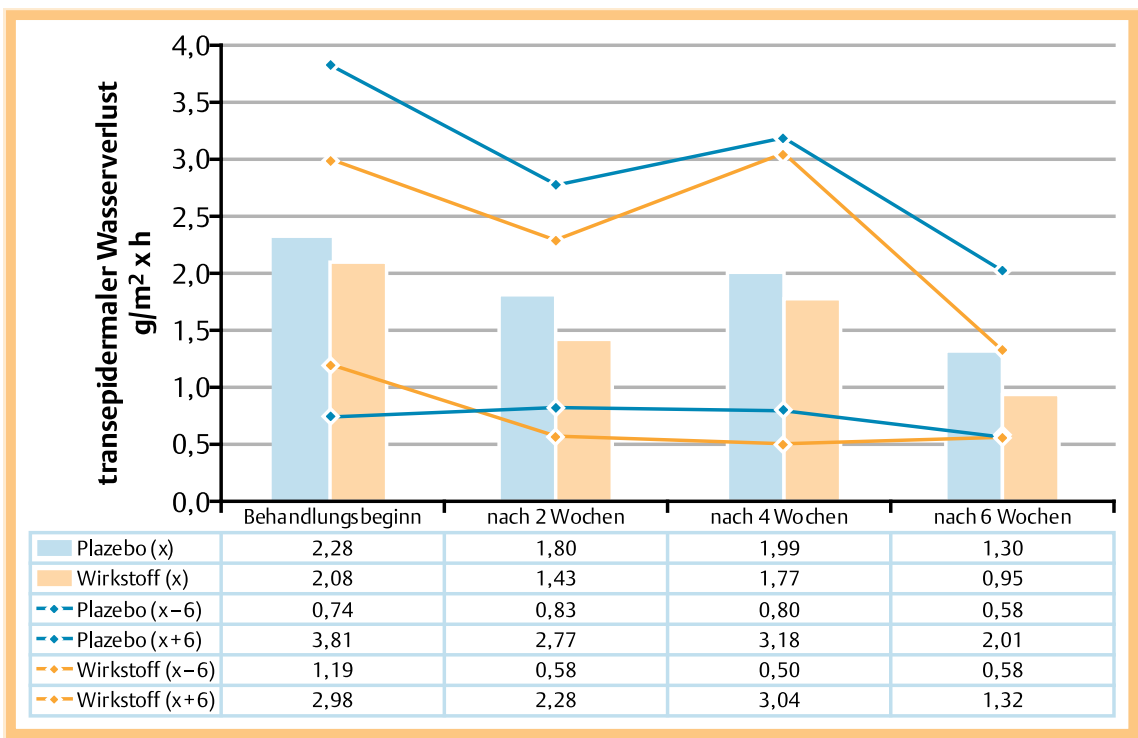

Abb. 6b Der Einfluss wirkstofffreier Salbe (Vehikel) und 2,5\%iger L-Arginin-Salbe (Wirkstoff) auf den transepidermalen Wasserverlust (TEWL) bei Hämodialysepatienten $(n=24)$. Veränderungen im Wochenvergleich (T-Test für Differenzen gegen Null): Woche 0, 2, 4 sowie 6: nicht signifikant.

\section{Diskussion}

$\nabla$

Pruritus und Hauttrockenheit - Harnstoffgehalt der Epidermis/Stratum corneum

Der urämische Pruritus wird auf nicht entzündlicher Haut bei terminaler Niereninsuffizienz häufig beobachtet $[8,9]$. Auch unter den Therapiemöglichkeiten der Hämo- und Peritonealdialyse ist der quälende urämische Pruritus zu 50-90\% vorhanden $[1,10,11]$. Wir fanden bei den 24 Hämodialysepatienten in $67 \%$ einen sehr starken und in 33\% einen mäßigen Pruritus (AtopieScore-Stufen 3 und 2 nach Diepgen [7]).

Kato et al. [11] gingen in einer Studie mit 50 Hämodialysepatienten der Frage des Schweregrades des Pruritus vor und nach der Dialyse nach. Der Juckreiz wurde anhand einer 100 Punkteskala klinisch bewertet. Es ließ sich keine Korrelation zwischen dem Ausmaß des Juckreizes und dem Alter und Geschlecht sowie der Dauer der Dialyse, den biochemischen Parametern, der zugrunde liegenden Nierenerkrankung und dem entzogenen Flüssigkeitsvolumen pro Dialyse feststellen. Bei den Dialysepatienten war die Hautfeuchtigkeit signifikant niedriger als bei den Kontrollpersonen, wobei es keine Korrelation zwischen der Ausprägung des Juckreizes und dem Hautfeuchtigkeitsgehalt gab.

Nur durch die Nierentransplantation mit suffizienter Nierenfunktion ist der urämische Pruritus zum Sistieren zu bringen. Parameter des Urämiesyndroms, wie der sekundäre Hyperparathyreoidismus (sHPT), begünstigen die Entstehung des Pruritus $[10,12-16]$. Ca. $2 / 3$ der hier vorgestellten Dialysepatienten hatten erhöhte Werte des iPTH (intaktes Parathormon) als Ausdruck des sHPT, bei ca. 1/3 lagen die Werte im Normbereich. Somit konnten wir bei den Hämodialysepatienten mindernde und fördernde Faktoren für den urämischen Pruritus feststellen, die aber keine Häufigkeitsveränderung im Auftreten des urämischen Pruritus erbrachten.

Die derzeitigen Therapieempfehlungen zum urämischen Pruritus beruhen auf der oralen Gabe von Aktivkohle, topischer Anwendung fetthaltiger Cremes sowie von Ölbädern und vorwiegend auf den 5 pathophysiologischen Hypothesen, die wir oben aufgezeigt haben: Überprüfen der Dialyseeffektivität, UVB-Bestrahlung, Elektroakupunktur, systemische Behandlung mit 
dem $\mu$-Rezeptor-Antagonisten Naltrexon und Applikation von Tacrolimussalbe [17-19].

Das Durchschnittsalter der Hämodialysepatienten lag bei 60 Jahren, das der Kontrollgruppe bei 56 Jahren. Somit waren für beide Untersuchungsgruppen zusätzlich die pathophysiologischen Veränderungen der Altershaut gegeben. Die Haut der Urämiker war, im Vergleich zur Haut der Kontrollpersonen (Altershaut), sehr trocken und stark juckend.

\section{Feuchthalte-Komplex-System der Haut und epidermaler} L-Argininstoffwechsel

Trockene Haut, u. a. bei Patienten mit atopischem Ekzem, jedoch auch bei der Altershaut, ist, wie seit Jahren bekannt, durch ein Harnstoffdefizit im Stratum corneum der Epidermis charakterisiert $[6,20]$. Harnstoff nimmt eine zentrale Rolle im „Feuchthalte-Komplex-System“ der Haut ein. Er ist einer der wichtigsten „natural moisturizing factors“ [21,22].

Werden diese Faktoren z. B. durch extensives Waschen der Haut aus dem Stratum corneum herausgelöst, verliert diese ihre Wasserbindungsfähigkeit. Sinkt die Feuchtigkeit im Stratum corneum auf Werte unter $8-10 \%$, wird die Haut trocken, rau und rissig. Die Moisturizer sind für die Wasserbindung in der Epidermis verantwortlich. Wenn aber, wie beim atopischen Ekzem, ein Harnstoffdefizit vorliegt, ist die Kausalität der Symptome erklärbar und möglicherweise ein Therapieansatz gegeben. Warum es jedoch zu einem Harnstoffdefizit kommt, ist ungeklärt [23]. Dialysepatienten haben, nach Donaubauer [23], jedoch erhöhte Harnstoffkonzentrationen nicht nur im Serum, sondern mit $37,2 \mu \mathrm{g} / 2,5 \mathrm{~cm}^{2}$ auch im Stratum corneum der Epidermis. Die Harnstoffkonzentration ist signifikant höher als bei Hautgesunden $\left(25,8 \mu \mathrm{g} / 2,5 \mathrm{~cm}^{2}\right)$. Das wirkt sich paradoxerweise nicht günstig auf den Wassergehalt der Epidermis bzw. auf die Feuchtigkeit der Haut der Hämodialysepatienten aus. Außerdem ist unklar, ob die erhöhte epidermale Harnstoffkonzentration Folge einer vermehrten Anflutung aus dem Blut ist oder ob nicht in den Keratinozyten selbst verstärkt Harnstoff gebildet wird.

Arginin zählt zu den glukoplastischen, nicht essenziellen Aminosäuren und wird vom Menschen endogen synthetisiert. Bei Extremsituationen (Schock, Sepsis, Wachstum) kann wegen eines Stickstoffdefizites die Synthese nicht ausreichend sein, sodass Arginin deshalb zu den „bedingt essenziellen“ Aminosäuren gezählt wird. Arginin liegt physiologisch in der optischen L-Konfiguration vor.

L-Arginin ist das Substrat mehrerer enzymatischer Reaktionen. Neben der Protein- und der Keratinsynthese ist Arginin auch das Substrat für die Arginase- und Stickstoffmonoxid (NO)-Synthase-Reaktion. Das Enzym Arginase setzt L-Arginin zu Harnstoff und L-Ornithin um. L-Ornithin kann wiederum im Harnstoffzyklus zu L-Citrullin metabolisiert werden. Das Enzym NOSynthase bildet aus L-Arginin NO und L-Citrullin. Beide Enzyme konkurrieren um das Substrat L-Arginin. Neben der Arginase Typ 1, der sog. hepatischen Form, gibt es eine zweite, extrahepatische Isoform, Typ 2 (A 2). Aus L-Arginin wird intraepidermal Harnstoff durch Keratinozyten neosynthetisiert. Diese exprimieren die L-isomerspezifische Arginase (A 2, Typ 2) in den Mitochondrien. Im Vergleich zu anderen Organen und Kompartimenten enthält die Epidermis den größten prozentualen Anteil an Arginase [24]. Wohlrab [24] konnte ein Konzentrationsmaximum der Arginase im Stratum basale und im unteren Stratum spinosum nachweisen. Diese Funktionseinheit bildet die germinative Zone des verhornenden Plattenepithels. In Differenzierungsrichtung kommt es zu einer funktionellen und morpholo- gischen Metamorphose der Keratinozyten bis hin zu den Korneozyten im Stratum corneum. L-Arginin kommt eine entscheidende Funktion bei der Bildung und Aufrechterhaltung der epidermalen Barrierefunktion zu. L-Arginin ist eine wichtige Harnstoffquelle und beeinflusst indirekt die Hydratation des Stratum corneum.

\section{Stickstoffmonoxid und trockene Haut sowie Pruritus}

Das Gas Stickstoffmonoxid (NO) hat eine Schlüsselstellung nicht nur im vegetativen Nervensystem, sondern auch in der Atherogenese im Sinn eines endogenen antiatherogenen Moleküls, letztlich über eine Regulationsfunktion im kardiovaskulären System, in der Makro- und in der Mikrostrombahn. NO wird durch die NO-Synthase (NOS) aus L-Arginin gebildet und rasch nach Oxygenierung zu Nitraten und Nitriten metabolisiert und damit inaktiviert. NOS wirkt im Epithel vermutlich im Sinne eines Radikalfängers und bei der immunologischen Signaltransduktion. Von besonderer Bedeutung ist die Funktion von NO bei der Auslösung und Unterhaltung von Entzündungsabläufen. NO ist für eine Maximierung der durch natürliche Killerzellen bzw. durch Lymphozyten-aktivierte Killerzellen bedingten Zytotoxizität verantwortlich und nimmt damit Einfluss auf tumor- und transplantationsimmunologische Entzündungsreaktionen. Zusätzlich reguliert NO die Freisetzung einer Reihe von Hormonen, besonders solche mit direkter oder indirekter Wirkung auf das kardiovaskuläre System [24].

Aktuell wurde gezeigt, dass in der Haut von TSK (tight-skin 1)-1/+ Mäusen, einem Tiermodell der Fibrose bzw. einem Sklerodermie-ähnlichen Syndrom, die endotheliale NOS in den Epidermisschichten, den Haarfollikeln und in den endothelialen Zellen entlang der Kapillaren nachweisbar ist [25]. Hervorzuheben ist, dass die Expression sowohl des endothelialen NOS-Proteins als auch des entsprechenden Gens in der Haut der TSK-1/+ Mäuse signifikant vermindert war.

Durch die Entdeckung der methylierten L-Arginin-Derivate symmetrisches (SDMA) und asymmetrisches Dimethylarginin (ADMA) konnte nachgewiesen werden, dass erhöhte ADMA-Konzentrationen NOS kompetitiv hemmen, indem ADMA anstelle von L-Arginin an NOS bindet, aber nicht zu NO umgesetzt wird. Auch bei Patienten mit chronisch terminaler Niereninsuffizienz und Hämodialyse wurden erhöhte ADMA-Konzentrationen nachgewiesen. Die erhöhten ADMA-Konzentrationen und die daraus resultierende endotheliale Dysfunktion erklären das hohe kardiovaskuläre Risiko von Dialysepatienten [26-29].

L-Arginin penetriert in die Haut des Menschen, wobei in der lebenden Epidermis ein ausreichendes Konzentrations-Zeit-Profil erreicht wird. Nach topischer Anwendung von L-Arginin-haltigen Präparationen konnten vasodilatative Effekte nachgewiesen werden $[24,30]$.

NO ist in der Altershaut vermindert, bedingt durch eine verstärkte Aktivität von reaktiven Sauerstoffspezies und/oder durch eine verminderte L-Arginin-Verfügbarkeit infolge hochregulierter Arginase-Aktivität [31]. Kürzlich zeigten Holowatz et al. [32], dass eine Erhöhung des L-Arginins als Substrat für die NO-Synthase entweder über eine Hemmung der Arginase oder direkt durch Arginin-Supplementierung erfolgt, und damit letztlich die altersbedingt verminderte kutane Vasodilatation verbessert und verstärkt wird. 
Einfluss von 2,5\%iger L-Argininhydrochlorid-haltiger Salbe auf die klinische Symptomatik des urämischen Pruritus

Die Dialysepatienten hatten folgenden Ausgangsbefund entsprechend dem Atopiescore [7]: Hauttrockenheit $88 \%$ - Stufe 3, Pruritus $67 \%$ - Stufe 3, Erythem $67 \%$ - Stufe 2, Schuppung 70\% Stufe 1. Bemerkenswerterweise war die Hauttrockenheit stärker ausgeprägt als der urämische Pruritus. Die Hauttrockenheit ließ sich bei den Dialysepatienten sowohl mit L-Arginin als auch mit wirkstofffreier Salbengrundlage signifikant bessern, deutlich wirksamer war jedoch L-Arginin.

Wohlrab [30] konnte durch topische Anwendung einer 10\%igen L-Arginin-haltigen Creme an gesunden Probanden eine Senkung des TEWL und eine Steigerung der Hydratation der Hornschicht erreichen, vermutlich beruhend auf einer Aktivierung der keratinozytären Arginase. L-Arginin ist eine wichtige Harnstoffquelle und hat eine solubilisierende Wirkung auf Proteine und trägt dadurch zusätzlich zur Steigerung der Wasserbindungskapazität der Hornschicht bei.

L-Arginin kann aus zytotoxikologischer Sicht als unbedenklich beurteilt werden. Die Anwendung von L-Arginin als Substrat der NOS-Isoformen in Keratinozyten und kutanen mikrovaskulären Endothelzellen hat, vermittelt durch die NO-Bildung, immunmodulatorische und antimikrobielle Wirkungen. Die Keimdichte auf der Haut und virale Superinfektionen bei trockener Haut werden durch Minderung der Keimadhärenz und immunmodulatorische Einflüsse erschwert.

Donaubauer [23] und Nenoff et al. [6] untersuchten den Einfluss von topisch applizierter 2,5\%iger Argininhydrochlorid-Salbe auf den Harnstoffgehalt des Stratum corneum und die Hautfeuchtigkeit bei atopischem Ekzem und Altershaut. Topisch zugeführtes Arginin stimuliert signifikant die Harnstoffsynthese der Hornhautzellen der Altershaut und zumindest temporär die der Haut bei atopischem Ekzem. Damit verbunden war ein Schwinden von Hauttrockenheit, Pruritus, Erythem und Schuppung. Die Autoren schlossen daraus, dass ein Harnstoffmangel bei Altershaut auf einer reduzierten Zufuhr bzw. Synthese und/oder einem erhöhten Verbrauch bzw. Abtransport beruhen kann.

Die Hauttrockenheit der Urämiker konnte von uns besonders durch die 2,5\%ige L-Argininhydrochlorid-Salbe, schwächer durch die wirkstofffreie Präparation signifikant gebessert werden. Dieses Ergebnis ist durch eine Erhöhung der Harnstoffkonzentration im Stratum corneum aus oben angegebenen Gründen nicht zu erklären. Vergleichende Untersuchungen an der urämischen Haut liegen nicht vor. Offenbar müssen andere Mechanismen in der Haut der Urämiker durch topische Applikation von 2,5\%iger L-Argininhydrochlorid-Salbe zur Wirkung kommen.

Der positive, aber geringere Effekt der wirkstofffreien Salbe ist offenbar auf den $47 \%$ igen Anteil von Vaseline zurückzuführen. Nach Tronnier [33] entsteht nach Auftragen einer Vaselineschicht über eine gewisse Zeit hinweg ein Wasserstau, der so lange anhält, bis diese Lipidschicht durch auf der Haut stehende kleine Wasserdepots gesprengt und durchbrochen wird.

Der urämische Pruritus konnte bei den Dialysepatienten mit der 2,5\%igen L-Argininhydrochlorid-Salbe signifikant gebessert werden. Selbst noch in Woche 6 war bei $87 \%$ der Patienten kein Pruritus mehr nachweisbar, bei $12 \%$ nur noch mit der Scorestufe 1. Mit der Vehikelpräparation war dieser Effekt auch signifikant, aber schwächer nachzuweisen.

Nach Bacharach-Buhles \& Altmeyer [34] konnten in der Haut der Urämiker mit chronischer Hämodialyse schwere Alterationen der kleinen korealen Gefäße nachgewiesen werden. Die Gefäß- wände waren auf Kosten des Lumens stark verdickt. Neuere Untersuchungen haben ergeben, dass NO in seiner Wirkung als ein antiarteriosklerotisches Molekül anzusehen ist. Bei arteriosklerotischen Risikofaktoren, Fettstoffwechselstörungen, Hypertonie, Hyperhomocysteinämie, sHPT, wie sie beim chronischen Urämiesyndrom vorliegen, kommt es zur Erhöhung des asymmetrischen Dimethylarginins (ADMA). Die ADMA-Erhöhung korreliert mit der endothelialen Dysfunktion. Die Akkumulation von ADMA bei der terminalen Niereninsuffizienz ist u.a. durch die fehlende Nierenausscheidung bedingt [26, 28,35-46].

Die Hemmung der NOS-Aktivität durch erhöhte ADMA-Spiegel lässt sich durch ein vermehrtes Substratangebot von L-Arginin durch kompetitives Verdrängen von ADMA an der NOS aufheben. Damit lässt sich erklären, warum L-Arginin die Endothelfunktion bei Patienten mit arteriosklerotischen Risikofaktoren bzw. vaskulären Erkrankungen verbessert. Es liegen Untersuchungen vor, die durch orale, intravenöse und intraarterielle Gabe von L-Arginin bei Hypercholesterinämie, koronarer Herzkrankheit, Hypertension, Diabetes mellitus, peripherer arterieller Verschlusskrankheit und chronischer Niereninsuffizienz (Dialysepatienten) eine Verbesserung der Durchblutung erzielen konnten. Bei den Dialysepatienten wurde eine Fluss-vermittelte Dilatation in der Arteria radialis und Arteria brachiales nachgewiesen [41].

L-Arginin könnte auch über seinen Metaboliten L-Agmatin vasodilatierend wirken [47]. L-Agmatin ist ein Agonist mit Clonidinähnlichen Wirkungen, d.h. es stimuliert zentrale $\alpha$-2-Adrenozeptoren. Das führt zur Senkung des peripheren Sympathikotonus und damit (indirekt) zu einer Vasodilatation. Somit zeigen diese Studien, dass durch gezielte Substitution von L-Arginin die Wirkung von ADMA aufgehoben oder abgeschwächt werden kann. Durch die erhöhten ADMA-Konzentrationen erklärt sich u. a. die endotheliale Dysfunktion bei terminal Niereninsuffizienten und damit das sehr hohe kardiovaskuläre Risiko der chronischen Dialysepatienten [41,48-54].

Nach topischer Applikation von L-Arginin-haltigen Präparationen konnte Wohlrab [24] durch Laser-Doppler-Fluxmetrie vasodilatative Effekte nachweisen, die er auf eine Aktivitätssteigerung der NOS-Isoformen zurückführte. Ein mikrovasodilatativer Wirkmechanismus von 2,5\%iger L-Argininhydrochlorid-Salbe auf den Rückgang von Hauttrockenheit und urämischen Pruritus lässt sich deshalb vermuten. Die Wirkung von 2,5\%iger L-Argininhydrochlorid-Salbe auf den Pruritus ist offenbar zusätzlich durch den immunmodulatorischen und antimikrobiellen Effekt von NO bedingt.

Auffallend waren die Ergebnisse des Wirkstoffes und der Vehikelpräparation auf die Symptome Erythem und Schuppung der Hämodialysepatienten. Diese Symptome waren gegenüber Hauttrockenheit und Pruritus deutlich schwächer ausgeprägt. Sie waren aber nur mit dem Wirkstoff signifikant zu bessern. Ursächlich ist weiterhin eine gesteigerte Penetration von L-Arginin in koreale Schichten bei pathologischen Hautzuständen nach Wohlrab $[24,30]$ anzunehmen.

Bei den Kontrollpersonen waren die Symptome Trockenheit, Pruritus, Erythem und Schuppung erheblich diskreter ausgeprägt, praktisch im Rahmen der Veränderungen bei der Altershaut. Zwischen Wirkstoff und Vehikel waren in der Wirkung auf Trockenheit und Pruritus keine signifikanten Unterschiede festzustellen, intensiver wirkte jedoch die 2,5\%ige L-Argininhydrochlorid-Salbe. Das entspricht prinzipiell den Ergebnissen von Nenoff et al. [6] zur Wirkung von 2,5\%iger L-Argininhydrochlorid-Salbe auf die Zunahme der Hautfeuchtigkeit und den 
Rückgang der Symptome bei der Altershaut, obwohl wir keine signifikanten Wirkungen feststellen konnten. Erythem und Schuppung waren bei den Kontrollpersonen so diskret ausgeprägt, dass sich keine nennenswerten Aussagen zwischen Wirkstoff und Vehikel ergaben.

\section{Einfluss von 2,5\% L-Argininhydrochlorid-Salbe} auf die Hautfeuchtigkeit und den transepidermalen Wasserverlust

Bei den Hämodialysepatienten wurde mit der Corneometrie in der Woche 0 (vor Therapie) ein Wert von $62,55 \mathrm{mg} / \mathrm{H}_{2} 0 / \mathrm{cm}^{2}$ gemessen, bei den Kontrollpersonen ein Wert von $68,14 \mathrm{mg} /$ $\mathrm{H}_{2} \mathrm{O} / \mathrm{cm}^{2}$. Somit konnten wir, ebenfalls wie andere Autoren $[11,55,56]$, eine deutlich erniedrigte Hautfeuchtigkeit bei den Hämodialysepatienten bestätigen. Unter der topischen Anwendung von L-Arginin konnte, gegenüber Vehikel, bei den Hämodialysepatienten die Hautfeuchtigkeit stärker angehoben werden. Auffallend waren die Messwerte in der Woche 6, nach Absetzen von Wirkstoff und Vehikel in Woche 4. Bei den Hämodialysepatienten wurde ein signifikant stärkerer Abfall der Hautfeuchtigkeit nach Absetzen von Wirkstoff gegenüber Vehikel gemessen. Somit muss ein Wirkmechanismus durch die 2,5\%ige L-Argininhydrochlorid-Salbe auf die Hydratation des Stratum corneum bei Urämikern angenommen werden, zusätzlich kommt die kurze Halbwertszeit von NO zur Wirkung.

Die Messwerte bei den Kontrollpersonen ergaben zwischen L-Arginin und Vehikel in der Zunahme der Hautfeuchtigkeit keinen signifikanten Unterschied, eine stärkere Wirkung war durch die 2,5\%iger L-Argininhydrochloridsalbe nachzuweisen. Die Wirkungen von Wirkstoff und Vehikel auf die Haut der Kontrollpersonen entsprechen im Prinzip, allerdings ohne Signifikanz, den Ergebnissen von Nenoff et al. [6] bei Applikation beider Präparationen auf die Altershaut.

Die Messungen des transepidermalen Wasserverlustes ergaben bei den Hämodialysepatienten zwischen topischer Applikation von L-Arginin und Vehikel keinen signifikanten Unterschied. Mit beiden Präparationen war eine Abnahme des TEWL festzustellen, nach Absetzen beider in Woche 4 ein tendenzieller Anstieg. Bei den Dialysepatienten war in Woche 0 (vor Therapie) der Wert des TEWL signifikant niedriger als der der Kontrollen $\left(1,27 \mathrm{~g} / \mathrm{m}^{2} \times \mathrm{h}\right.$ vs. $\left.2,08 \mathrm{~g} / \mathrm{m}^{2} \times \mathrm{h}\right)$, dem entspricht die niedrige Hautfeuchtigkeit der Urämiker im Vergleich zu den Kontrollen. Auffallend war auch, dass der TEWL bei Dialysepatienten durch Vehikel signifikant vermindert werden konnte. Bei den Kontrollpersonen ergaben sich zwischen L-Arginin und Vehikel keine signifikanten Unterschiede des TEWL. Das entspricht den Befunden von Nenoff et al. [6] an der Altershaut.

Keine unerwünschten Nebenwirkungen unter topischer L-Argininhydrochlorid-Behandlung

Hervorzuheben ist, dass während des sechswöchigen Untersuchungsablaufes mit der 2,5\%igen L-Argininhydrochlorid-Salbe und der wirkstofffreien Salbe keine kutanen Nebenwirkungen (Brennen, progrediente Rötung, kontaktallergische Reaktionen, irritativ-subtoxische Reaktionen) bei beiden Untersuchungsgruppen festgestellt werden konnten, entsprechend den Erfahrungen der Studie von Nenoff et al. [6].

\section{Fazit für die Praxis}

$\nabla$

Es ist gelungen, durch topische Applikation von 2,5\%iger L-Argininhydrochlorid-Salbe den urämischen Pruritus und die Hauttrockenheit, außerdem Erythem und Schuppung bei Hämodialysepatienten signifikant zu bessern [57]. L-Arginin wird bei pathologisch veränderter Haut, also auch bei Dialysepatienten, besser resorbiert, sodass ein vermehrtes Substratangebot von L-Arginin entsteht. Bei der terminalen Niereninsuffizienz liegen erhöhte ADMA (asymmetrisches Dimethylarginin)-Spiegel vor, die eine endotheliale Dysfunktion bewirken. Durch vermehrte Applikation von L-Arginin (topisch, jedoch auch systemisch) kommt es zum kompetitiven Verdrängen von ADMA an den NOS-Isoformen und damit werden vasodilatative Wirkungen von NO erzielt. Durch die topische Applikation von L-Arginin als Substrat der NOS-Isoformen lassen sich zudem immunmodulatorische und antimikrobielle Eigenschaften von NO zur Wirkung bringen. Die topische Gabe von L-Arginin lässt dessen solubilisierende Wirkung auf Proteine zur Geltung kommen und damit wird die Wasserbindungskapazität der Hornschicht gesteigert.

Die positiven, aber geringeren Effekte nach topischer Applikation einer wirkstofffreien Salbe auf die urämischen Hautveränderungen sind auf deren hohen Anteil von Vaseline (47\%) mit einem hydratationsfördernden Effekt zurückzuführen. Die positiven Wirkungen beider Präparationen - L-Arginin und wirkstofffreie Vehikelpräparation - auf die Altershaut der gesunden Kontrollpersonen entsprechen den Ergebnissen der Literatur, indem die altersbedingt niedrige Harnstoffkonzentration im Stratum corneum der Epidermis erhöht wird.

\section{Abstract}

Topical L-Arginine Hydrochloride Ointment Improves Skin Dryness and Pruritus in Patients under Chronic Haemodialysis - A Vehicle-Controlled Prospective Randomised Study $\nabla$

Background: The uraemic pruritus is a very painful symptom affecting haemodialysis patients and is observed in 50 to $90 \%$ of patients. The etiology of uraemic pruritus have not yet been clarified. Only after kidney transplantation with a functioning transplant the uraemic pruritus is stopped. This is why no causal therapy exists so far.

Patients and methods: We examined in 24 chronic dialysis patients over six weeks the topical effect of a $2.5 \% \mathrm{~L}$-arginine hydrochloride ointment (week 1 to 4 ) in comparison to an active agent free ointment for skin dryness, pruritus, erythema and desquamation. The control group consisted of 12 subjects of healthy skin and kidneys.

Results: Skin hydration and the pruritus of haemodialysis patients could be significantly improved by the active agent and the vehicle preparations with the active agent being clearly more effective. An improvement of erythema and desquamation could only be achieved by the active agent preparation. The values measured by corneometry showed a stronger increase of skin hydration through the active agent preparation. The values measured by tewametry showed no difference between active agent and vehicle preparation.

Conclusions: The positive effect of $2.5 \% \mathrm{~L}$-arginine hydrochloride ointment on uraemic pruritus can be theoretically explained 
insofar that L-arginine as a substrate of NOS isoforms prompts the immunomodulatory, anti-microbial and vasodilative properties of NO to become effective.

\section{Literatur}

1 Ulrich M, Stockfleth E. Hauterkrankungen bei Dialyse- sowie organtransplantierten Patienten. Nieren- und Hochdruckkr 2006; 35: $103-109$

2 Wikström B. Itchy skin - a clinical problem for haemodialysis patients. Nephrol Dial Transplant 2007; 22, Suppl 5: 3-7

3 Mügge C, Sandholzer H. Juckreiz - der kleine Bruder des Schmerzes? MMW 2008; im Druck

4 Durrant-Finn U, Osten B, Mügge C, Nenoff P. Pruritus und Hauttrockenheit bei chronischer Niereninsuffizienz und Hämodialysepatienten eine Übersicht. Wiener Med Wochenschr, im Druck

5 Yosipovitch G, Duque MI, Patel TS, Ishiuji Y, Guzman-Sanchez DA, Dawn $A G$, Freedman BI, Chan YH, Crumrine D, Elias PM. Skin barrier structure and function and their relationship to pruritus in end-stage renal disease. Nephrol Dial Transplant 2007; 22: 3268 - 3272

6 Nenoff P, Donaubauer K, Arndt T, Haustein U-F. Topisch appliziertes Agininhydrochlorid. Einfluss auf den Harnstoffgehalt des Stratum corneum und die Hautfeuchtigkeit bei atopischem Ekzem und Altershaut. Hautarzt 2004; 55: 58-64

7 Diepgen TL, Fartasch M, Hornstein OP. Kriterien zur Beurteilung der atopischen Hautdiathese. Dermatosen Beruf Umwelt 1991; 39: 7983

8 Ständer S, Weisshaar E. Chronischer Pruritus. Dtsch Arztebl 2005; 102 : $2553-2559$

9 Pusl T, Beuers U. Leitsymptom Juckreiz. Dtsch Ärztebl 2006; 103: $1249-1256$

10 Stahle-Bäckdahl M, Hägermark Ö, Lins L-E, Törring $O$, Hilliges $M$, Johansson 0 . Experimental and immunhistochemical studies on the possible role of parathyroid hormone in uraemic pruritus. J Internal Med 1989; 225: 411 - 415

11 Kato A, Hamada M, Maruyama T, Maruyama Y, Hishida A. Pruritus and hydration state of stratum corneum in hemodialysis patients. Am J Nephrol 2000; 20: 437-442

12 Hampers CL, Katz AI, Wilson RE, Merrill JP. Disappearance of uremic itching after subtotal parathyroidectomy. N Engl J Med 1968; 279: $695-697$

13 Altmeyer $P$, Kachel $H-G$, Jünger $M$, Koch K-M, Holzmann $H$. Hautveränderungen bei Langzeitdialysepatienten. Hautarzt 1982; 33: 303-309

14 Denman ST. Continuing medical education. A review of pruritus. J Am Acad Dermatol 1986; 14: 375 - 392

15 Ponticelli C, Bencini PL. Uremic pruritus: A review. Nephron 1992; 60: $1-5$

16 Chou F-F, Ho J-Ch, Huang S-C, Sheen-Chen S-M. A study on pruritus after parathyreoidectomy for secondary hyperparathyreoidism. J Am Coll Surg 2000; 190: 65 - 70

17 Mettang T. Urämischer Pruritus - Neue Sichtweise eines alten Problems. Spektr Nephrol 2003; 1: 3-10

18 Mettang T, Kuhlmann U. Chronische Niereninsuffizienz. In: Kuhlmann U, Walb D, Luft FC (Hrsg). Nephrologie. Stuttgart: Georg Thieme Verlag, 2003: $306-370$

19 Ständer S. Rationelle symptomatische Therapie bei chronischem Pruritus. Hautarzt 2006; 57: $403-410$

20 Wellner K, Wohlrab W. Quantitative evaluation of urea in stratum corneum of human skin. Arch Dermatol Res 1993; 285: 239-240

21 Wohlrab W. Der Einfluss von Harnstoff auf die Wasserbindungskapazität der menschlichen Hornschicht. Dermatol Mon-schr 1988; 174: $622-627$

22 Wohlrab W. Einfluss des Harnstoffgehaltes unterschiedlicher Emulsionen auf die Wasserbindungskapazität der menschlichen Hornschicht. Z Hautk 1990; 66: 390-395

23 Donaubauer K. Untersuchungen zum Harnstoffgehalt des Stratum corneum der Epidermis bei Dermatologischen Erkrankungen sowie unter dem Einfluss von aminosäurehaltigen Externa. Dissertation, Medizinische Fakultät der Universität Leipzig 2002

24 Wohlrab J. Der epidermale Arginin-Stoffwechsel und dessen therapeutische Beeinflussung. Akt Dermatol 2002; 28: 13-20

25 Dooley A, Low SY, Holmes A, Kidane AG, Abraham DJ, Black CM, Bruckdorfer $K R$. Nitric oxide synthase expression and activity in the tight- skin mouse model of fibrosis. Rheumatology (Oxford) 2008; 47 $272-280$

26 Kielstein JT, Fliser D. Asymmetrisches Dimethylarginin (ADMA) - ein kleines Molekül mit großer Bedeutung für den Nephrologen? NephroNews 2003; 5: 2-5

27 Sunder-Plaßmann G, Hörl WH. Urämietoxine. In: Hörl WH, Wanner C (Hrsg). Dialyseverfahren in Klinik und Praxis. Stuttgart: Georg Thieme Verlag, 2004: 397-401

28 Fliser D. Asymmetrisches Dimethylarginin (ADMA). Nephro-News 2004; 3: 23

29 Brodde O-E. Die Bedeutung von L-Arginin und NO für die Behandlung der endothelialen Dysfunktion. Nieren- und Hochdruckkrankheiten 2004; 33: 679-686

30 Wohlrab J, Siemens Ch, Marsch WC. The influence of L-arginine on the regulation of epidermal arginase. Skin Pharmacol Appl Skin Physiol 2002; $15: 44-45$

31 Holowatz LA, Thompson CS, Kenney WL. Acute ascorbate supplementation alone or combined with arginase inhibition augments reflex cutaneous vasodilation in aged human skin. Am J Physiol Heart Circ Physiol 2006; 291: H2965-H2970

32 Holowatz LA, Thompson CS, Kenney WL. L-Arginine supplementation or arginase inhibition augments reflex cutaneous vasodilatation in aged human skin. J Physiol 2006; 574: 573-581

33 Tronnier M. Hydratation der Haut. Ärztl Kosmetol 1984; 14: 365 - 375

34 Bacharach-Buhles M, Altmeyer P. Hauterkrankungen. In: Hörl WH, Wanner C (Hrsg). Dialyseverfahren in Klinik und Praxis. Stuttgart, New York: Georg Thieme Verlag, 2004: 534-540

35 Palmer RMJ, Rees DD, Asthton DS, Moncada S. L-arginine is the physiological precursor for the formation of nitric oxide in endothelium-dependent relaxation. Biochem Biophys Res Comm 1988; 153: 1251 1256

36 Mori M, Gotoh T. Regulation of nitric oxide production by arginine metabolic enzymes. Biochem Biophys Res Comm 2000; 275: 715 - 719

37 Böger RH, Bode-Böger SM. The clinical pharmacology of L-arginine Annu Rev Pharmacol Toxicol 2001; 41: 79-99

38 Zoccali C, Bode-Böger M, Mallamaci F, Benedetto FA, Tripepi G, Malatino LS, Cataliotti A, Bellanuova I, Fermo I, Frölich JC, Böger RH. Plasma concentration of asymmetrical dimethylarginine and mortality in patients with end-stage renal disease: a prospective study. Lancet 2001; 358: $2113-2117$

39 Xiao S, Wagner L, Mahaney J, Baylis Ch. Uremic levels of urea inhibit L-arginine transport in cultured endothelial cells. Am J Physiol Renal Physiol 2001; 280: 989-995

40 Berkowitz DE, White R, Li D, Minhas KM, Cernetich A, Kim S, Burke S, Shoukas AA, Nyhan D, Champion HC, Hare JM. Arginase Reciprocally Regulates Nitric Oxide Synthase, Activity and Contributes to Endothelial Dysfunction in Aging Blood Vessels. Circulation 2003; 108: 2000 2006

41 Delles Ch, Schneider M, Oehmer S, Fleischmann EH, Schmieder RE. L-arginine-induced vasodilatation of the renal vasculature is not altered in hypertensive patients with type 2 diabetes. Diab Care 2003; 26 : $1836-1840$

42 Böger RH. Asymmetrisches Diethylarginin (ADMA) als kardiovaskulärer Risikofaktor. Dtsch Med Wochenschr 2001; 129: 820 - 824

43 Sunder-Plaßmann G, Hörl WH. Urämietoxine. In: Hörl WH, Wanner C (Hrsg). Dialyseverfahren in Klinik und Praxis. Stuttgart: Georg Thieme Verlag, 2004: $397-401$

44 Brodde O-E, Philipp T. Bedeutung des L-Arginin/NO-Systems für die Wirkung von Nebivolol. Dtsch Med Wochenschr 2006; 131: 2545 2550

45 Bode-Böger SM. Einfluss von L-Arginin auf die Arterioskleroseentwicklung: Was ist therapeutisch gesichert? Dtsch Med Wochenschr 2005; 130: $593-598$

46 Galle J. Lipide und endotheliale Dysfunktion - Kardiovaskuläre Komplikationen bei chronischer Niereninsuffizienz. Mitteilungen der Deutschen Arbeitsgemeinschaft für Klinische Nephrologie 2005: 107- 111

47 Wohlrab J. L-Arginin oder Agmatin? Hautarzt 2004; 55: 689-691

48 Vogel RM, Sonntag M, Deussen A. Einfluss der Arginin-abhängigen Stickoxidsynthese auf die regionale Durchblutung des Auges am narkotisierten Hund. Ophthalmologe 1994; 91: 763 - 767

49 Böger RH, Bode-Böger SM, Thiele W, Creutzig A, Alexander K, Frölich JC. Restoring vascular nitric oxide formation by L-arginine improves the symptoms of intermittent claudication in patients with peripheral arterial occlusive disease. JACC 1998; 32: 1336- 1344 
50 Lerman A, Burnett JC, Higano ST, McKinley LJ, Holmes DR. Long-term L-arginine supplementation improves small-vessel coronary endothelial function in humans. Circulation 1998; 97: 2123-2128

51 Lekakis JP, Papathanassiou S, Papaioannou TG, Papamichael CM, Zakopoulos N, Kotsis V, Dagre AG, Stamatelopoulos K, Protogerou A, Stamatelopoulos SF. Oral L-arginine improves endothelial dysfunction in patients with essential hypertension. Int J Cardiol 2002; 86: 317 - 323

52 Niebauer J, Maxwell AJ, Lin PS, Wang D, Tsao PS, Cooke JP. NOS inhibition accelerates atherogenesis: reversal by exercise. Am J Physiol Heart Circ Physiol 2003; 285: 535- 540

53 Bode-Böger StM, Muke J, Surdacki A, Brabant G, Böger RH, Frölich J. Oral L-arginine improves endothelial function in healthy individuals older than 70 years. Vasc Med 2003; 8: 77-81
54 Piatti PM, Fragasso G, Monti LD, Setola E, Lucotti P, Fermo I, Paroni R, Galluccio E, Pozza G, Chierchia S, Margonato A. Acute intravenous L-arginine infusion decreases endothelin-1 levels and improves endothelial function in patients with angina pectoris and normal coronary arteriograms. Circulation 2003; 107: 429-436

55 Stahle-Bäckdahl $M$. Stratum corneum hydration in patients undergoing maintenance hemodialysis. Actra Derm Venereol (Stockh) 1988; 68: 531 - 544

56 Yosipovitch G, Tur E, Morduchowicz G, Boner G. Skin surface pH, moisture, and pruritus in haemodialysis patients. Nephrol Dial Transplant 1993; 8: 1129-1132

57 Durrant-Finn $U$. Der Einfluss von topisch appliziertem L-Arginin auf Trockenheit und Juckreiz der Haut bei Hämodialysepatienten in vivo. Dissertation, Universität Halle (Saale) 2008 